\title{
Propagation and neutrino oscillations in the base of a highly magnetized gamma-ray burst fireball flow
}

\author{
N. Fraija ${ }^{1}$ \\ Instituto de Astronomía, UNAM, México, 04510 \\ nifraija@astro.unam.mx
}

Received __ ; accepted _

\footnotetext{
${ }^{1}$ Luc Binette-Fundación UNAM Fellow. Instituto de Astronomía, Universidad Nacional Autónoma de México, Circuito Exterior, C.U., A. Postal 70-264, 04510 México D.F., México
} 


\begin{abstract}
Neutrons play an important role in the dynamics of gamma-ray bursts. The presence of neutrons in the baryon-loaded fireball is expected. If the neutrons abundance is comparable to that of protons, important features may be observed such as quasithermal multi-GeV neutrinos in coincidence with a subphotospheric $\gamma$-ray emission, nucleosynthesis at later times and rebrightening of the afterglow emission. Additionally, thermal $\mathrm{MeV}$ neutrinos are created by electron-positron annihilation, electron (positron) capture on protons (neutrons) and nucleonic bremsstrahlung. Although $\mathrm{MeV}$ neutrinos are difficult to detect, quasi-thermal $\mathrm{GeV}$ neutrinos are expected in $\mathrm{km}^{3}$ detectors and/or DeepCore+IceCube. In this paper, we show that neutrino oscillations have outstanding implications for the dynamics of the fireball evolution and also that they can be detected through their flavor ratio on Earth. For that we derive the resonance and charged-neutrality conditions as well as the neutrino self-energy and effective potential up to order $m_{W}^{-4}$ at strong, moderate and weak magnetic field approximation to constrain the dynamics of the fireball. We found important implications: i) resonant oscillations are suppressed for high baryon densities as well as neutrons abundance larger than that of protons and ii) the effect of magnetic field is to decrease the proton-to-neutron ratio aside from the number of multi-GeV neutrinos expected in DeepCore detector. Also we estimate the GeV neutrino flavor ratios along the jet and on Earth.
\end{abstract}

Subject headings: Physical Data and Processes: magnetic fields — Physical Data and Processes: neutrinos — Stars:gamma-ray burst: general — stars: jets 


\section{Introduction}

Gamma-ray bursts (GRBs) are brief events occurring at an average rate of some days throughout the universe. These short, energetic bursts of gamma-rays mark the most violent, cataclysmic explosions in the universe, likely associated with the birth of stellar-size black-holes or rapidly spinning, highly magnetized neutron stars. One of the most successful theory in terms of explaining GRBs and their afterglows is the fireball model (see Mészáros 2006; Zhang \& Mészáros 2004, for recent reviews). This model predicts an expanding ultrarelativistic shell that moves into the external surrounding medium. The collision of the expanding shell with another shell (internal shocks) or the interstellar medium (external shocks) gives rise to radiation emission through the leptonic (synchrotron and synchrotron self-Compton (SSC) radiation) or hadronic (photo-pion decay and inelastic proton-neutron collision) processes. In addition, when the expanding relativistic shell encounters the external medium two shocks are involved: an outgoing, or forward shock (Rees \& Mészáros 1994; Paczyński \& Rhoads 1993) and another one that propagates back into the ejecta, the reverse shock (Mészáros \& Rees 1994, 1997a). The base of the fireball flow is connected to the GRB central engine, a black hole (BH) - torus system or a rapidly rotating magnetar. It is endowed with magnetic fields and formed by free

nucleons, $e^{ \pm}$pairs in thermal equilibrium of $1-10 \mathrm{MeV}$ inside the initial scale $\sim 10^{7} \mathrm{~cm}$ assuming a $10 M_{\odot} \mathrm{BH}$. In the initial state, the baryons are essentially at rest with respect to the central engine (Zhang \& Mészáros 2004).

In recent years, two observational facts have supported the idea that the central engine could be magnetized. i) The strong gamma-ray polarization (Coburn and Boggs 2003; Boggs \& Coburn 2003; Rutledge \& Fox 2004) and ii) a stronger magnetic field in reverse than in forward shock (magnetization of the jet) as a result of a full description of GRBs 980923, 990123, 021004, 021211, 041219A , 090926A and others (Zhang \& Meszaros 2002; Zhang et al. 2003a; Fan et al. 2005; Fraija et al. 2012a b; Sacahui et al. 2012), so that the fireball may be endowed with a primordial magnetic field (Zhang et al. 2003a; Zhang \& Kobayashi|2005). Recently, a relevant 
result based on the magnetization of outflow and the non detection of neutrinos from GRBs by IceCube (Abbasi \& et al. 2012b) was found by Zhang \& Kumar (2013). They proposed that the suppression of the neutrino flux in the gamma-ray emission region is a consequence of the degree of magnetization of the outflow.

The evolution of the fireball with magnetic fields has been explored by various authors (Usov 1999; Wheeler et al. 2000; Blandford 2002; Lyutikov \& Blandford 2002; Spruit \& Drenkhahn 2003; Vlahakis and Königl 2003a b; Zhang \& Kumar 2011). In these models an electromagnetic component is introduced in the dynamics of the standard fireball. This extra component is taken into account through the magnetization parameter $(\sigma)$, defined as the ratio of Poynting flux (electromagnetic component) to matter energy (internal+kinetic component). Depending on the dissipation process, a part of the dissipated magnetic energy is converted into internal energy, and the other part is used to accelerate the fireball (Michel 1969; Mészáros 2012).

The energy requirement (isotropy-equivalent luminosities $L_{\gamma} \geq 10^{52} \mathrm{erg} \mathrm{s}^{-1}$ ) demands magnetic fields at the base in excess of $B \sim 10^{15} \mathrm{G}$, that can be produced by shear and instabilities in an accreting torus around the BH. The energy source can be either the accretion energy or via magnetic coupling between the disk and $\mathrm{BH}$, extraction of angular momentum from the latter occurring via the Blandford-Znajek mechanism (Blandford \& Znajek 1977; Mészáros \& Rees 1997b; Usov 1992; Lee et al.2000; Putten et al. 2001).

On the other hand, the presence of neutrons and their abundance in the fireball GRB plays an important role in the dynamics of the jet and its relation with the observed photon and neutrino flux emission (Koers \& Giannios 2007; Metzger et al. 2008; Razzaque \& Meszaros 2006a; Dermer \& Atoyan 2006). Among the more relevant consequences are: i) generation of quasi-thermal GeV neutrinos and subphotospheric $\gamma$-ray emission via inelastic collisions between streaming protons and neutrons in the fireball. Above the pion production threshold $(\sim 140 \mathrm{MeV})$, the n-p decoupling is followed by inelastic n-p scattering leading to $\pi^{ \pm}$and $\pi^{0}$. As known, $\pi^{0}$ decays to two photons $(\gamma+\gamma)$ and $\pi^{+}$to four particles (three neutrinos) $\left(\mu^{+}+\nu_{\mu}\right.$ and then $\mu^{+}$to $\left.e^{+}+\nu_{e}+\bar{\nu}_{\mu}\right)$, which 
is the first signature in photons and neutrinos (Derishev,et al. 1999; Bahcall \& meszaros 2000; Meszaros \& Rees 2000; Razzaque \& Meszaros 2006b; Murase et al. 2013), ii) the effects on the development, strength and energy range of internal shocks (Rossi et al. 2006; Xue et al. 2008; Fan et al. 2005; Fan \& Wei 2004), iii) nucleosyntesis (Metzger et al. 2008; Beloborodov 2003a), and iv) rebrightening of the afterglow emission. Neutrons decay after $881.5 \mathrm{~s}$, hence they play an important role at large radii $\sim 10^{16}-10^{17} \mathrm{~cm}$ where external shocks happen as even an exponential small number of survival neutrons carry an energy much larger than the rest-energy of circumburst medium (Derishev,et al. 1999; Beloborodov 2003a b; Rossi et al. 2006). Furthermore, in the initial stage and during the expansion of the fireball thermal neutrinos will be produced by electron-positron annihilation $\left(e^{+}+e^{-} \rightarrow Z \rightarrow \nu_{j}+\bar{\nu}_{j}\right)$, processes of electron capture on protons $\left(e^{-}+p \rightarrow n+\nu_{e}\right)$ and positron capture on neutrons $\left(e^{+}+n \rightarrow p+\overline{\nu_{e}}\right)$, and nucleon-nucleon bremsstrahlung $\left(N N \rightarrow N N+\nu_{j}+\bar{\nu}_{j}\right)$ for $j=e, \nu, \tau$. Also neutrinos of similar energies are naturally expected in the accretion disk during the collapse or merger. As known, properties of neutrinos get modified when they propagate in this magnetized fireball and, depending on the neutrino flavor, would feel a different effective potential because electron neutrino $\left(\nu_{e}\right)$ interacts with electrons via both neutral and charged currents $(\mathrm{CC})$, whereas muon $\left(\nu_{\mu}\right)$ and tau $\left(\nu_{\tau}\right)$ neutrinos interact only via the neutral current (NC). This would induce a coherent effect in which maximal conversion of $\nu_{e}$ into $\nu_{\mu}\left(\nu_{\tau}\right)$ takes place even for a small intrinsic mixing angle (Wolfenstein 1978). Although thermal neutrino oscillations have been widely studied in the literature for different scenarios (Ruffert \& Janka 1999, Goodman et al. 1987; Volkas \&

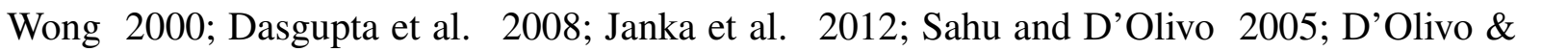
\begin{tabular}{|l|l|l|l||l|}
\hline Nieves & $1996 b ;$ & Erdas \& Isola 2000; D’Olivo \& Nieves 1997; D’Olivo et al. 2003 ; Erdas \\
\hline
\end{tabular} 2009; Sahu et al. 2009a b), and even for $\mathrm{O}\left(\mathrm{m}_{W}^{-4}\right)$ corrections to the neutrino dispersion relation (Nötzold \& Raffelt 1988; Enquist et al. 1991; D'Olivo et al. 1992), a full analysis of both the thermal and subphotospheric neutrino oscillations over the dynamics of the fireball GRB as neutrons abundance is comparable to that of protons and the magnetic field is as strong as the 
critical magnetic field has not been performed. In this paper, we show that neutrino oscillations have important implications for the evolution of the fireball when it is highly magnetized and has a considerable amount of neutrons and protons. Firstly, we compute the neutrino self-energy by using real time formalism of finite temperature field theory (Nieves 1994; Weldon 1982;

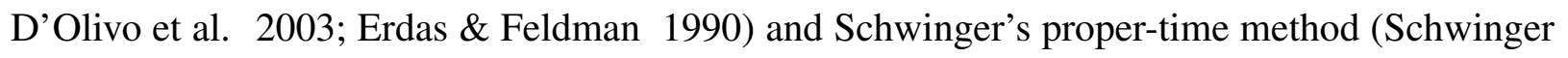
|1951). Then, taking into account the strong, moderate and weak magnetic field approximation, we compute, analyze and compare the neutrino effective potential up to order $\mathrm{m}_{W}^{-4}$ at all the regimes. Next, we derive the resonance conditions to find the range of values for which $\mathrm{MeV}$ - GeV neutrinos oscillate resonantly and then we do a full analysis of two- (solar, atmospheric and accelerator parameters) and three-neutrino mixing, even though we estimate the neutrino flavor ratio in the jet and on Earth. After that, we derive the charged-neutrality condition so that together with the resonance condition we can constrain the baryon density as well as the protons and neutrons abundance in the dynamics and evolution of a magnetized fireball. The results are also discussed in terms of magnetization degree.

We hereafter use $Q_{x} \equiv Q / 10^{x}$ in cgs units and $c=\hbar=\mathrm{k}=1$ in natural units

\section{Neutrino Effective Potential}

We use the finite temperature field theory formalism to study the effect of heat bath on the propagation of elementary particles (D'Olivo \& Nieves 1996a; Tututi et al. 2002). The effect of magnetic field is taken into account through Schwinger's propertime method (Schwinger 1951). The effective potential of a particle is calculated from the real part of its self-energy diagram. The neutrino field equation in a magnetized medium is

$$
[k-\Sigma(k)] \Psi_{L}=0
$$

where the neutrino self-energy operator $\Sigma(k)$ is a Lorentz scalar which depends on the characterized parameters of the medium, for instance, chemical potential, particle density, 
temperature, magnetic field, etc. For our purpose, $\Sigma(k)$ can be formed by

$$
\Sigma(k)=\mathcal{R}\left(a_{\|} k_{\|}+a_{\perp} k_{\perp}+b h+c b\right) \mathcal{L},
$$

where $k_{\|}^{\mu}=\left(k^{0}, k^{3}\right), k_{\perp}^{\mu}=\left(k^{1}, k^{2}\right)$ and $u^{\mu}$ stands for the 4-velocity of the center-of-mass of the medium given by $u^{\mu}=(1,0)$. The projection operators are conventionally defined as $\mathcal{R}=\frac{1}{2}\left(1+\gamma_{5}\right)$ and $\mathcal{L}=\frac{1}{2}\left(1-\gamma_{5}\right)$. The effect of the magnetic field enters through the 4 -vector $b^{\mu}$ which is given by $b^{\mu}=(0, \hat{\mathbf{b}})$. The background classical magnetic field vector is along the $z$-axis and consequently $b^{\mu}=(0,0,0,1)$. So using the four vectors $u^{\mu}$ and $b^{\mu}$ we can express

$$
k_{\|}=k_{0} h-k_{3} b,
$$

and the self-energy can be expressed in terms of three independent four-vectors $k_{\perp}^{\mu}, u^{\mu}$ and $b^{\mu}$. Therefore we can write $(\Sigma=\mathcal{R} \tilde{\Sigma} \mathcal{L})$

$$
\tilde{\Sigma}=a_{\perp} k_{\perp}+b h+c b
$$

The neutrino self-energy in a magnetic background can be found from Eq. (1)

$$
\operatorname{det}[k-\Sigma(k)]=0 .
$$

Using the Dirac algebra, the dispersion relation, $V_{\text {eff }}=k_{0}-|\mathbf{k}|$, as a function of Lorentz scalars can be written as

$$
V_{e f f}=b-c \cos \phi-a_{\perp}|\mathbf{k}| \sin ^{2} \phi,
$$

where $\phi$ is the angle between the neutrino momentum and the magnetic field vector. Now the Lorentz scalars $a, b$ and $c$ which are functions of neutrino energy, momentum and magnetic field can be calculated from the neutrino self-energy due to $\mathrm{CC}$ and $\mathrm{NC}$ interactions of neutrino with the background particles. 


\subsection{One-loop neutrino self-energy}

Let us consider one-loop corrections to the neutrino self-energy in the presence of a magnetic field. The one-loop neutrino self-energy comes from three pieces (Bravo and Sahu 2007; Elizalde et al. 2004; Erdas et al. 1998; Sahu et al. 2009a b), one coming from the $W$-exchange diagram which we will call $\Sigma_{W}(k)$ (Fig. 1(a)), one from the $Z$-exchange diagram which will be denoted by $\Sigma_{Z}(k)$ (Fig. 1(b)) and one from the tadpole diagram which we will designate by $\Sigma_{t}(k)$ (Fig. 1(c)). The total neutrino self-energy in a magnetized medium then becomes

$$
\Sigma(k)=\Sigma_{W}(k)+\Sigma_{Z}(k)+\Sigma_{t}(k) .
$$

The W-exchange diagram for the one-loop self-energy is

$$
-i \Sigma_{W}(k)=\mathcal{R}\left[\int \frac{d^{4} p}{(2 \pi)^{4}}\left(\frac{-i g}{\sqrt{2}}\right) \gamma_{\mu} i S_{\ell}(p)\left(\frac{-i g}{\sqrt{2}}\right) \gamma_{\nu} i W^{\mu \nu}(q)\right] \mathcal{L},
$$

where $g^{2}=4 \sqrt{2} G_{F} m_{W}^{2}$ is the weak coupling constant, $W^{\mu \nu}$ depicts the W-boson propagator which in the $\mathrm{eB} \ll \mathrm{m}_{W}^{2}$ limit and in unitary gauge is given by (Erdas et al. 1998; Sahu et al. 2009b)

$$
W^{\mu \nu}(q)=\frac{g^{\mu \nu}}{m_{W}^{2}}\left(1+\frac{q^{2}}{m_{W}^{2}}\right)-\frac{q^{\mu} q^{\nu}}{M_{W}^{4}}+\frac{3 i e}{2 m_{W}^{4}} F^{\mu \nu}
$$

here $\mathrm{m}_{W}$ is the W-boson mass, $g^{\mu \nu}$ is the metric tensor and $F^{\mu \nu}$ is the electromagnetic field tensor. $S_{l}(\mathrm{p})$ stands for the charged lepton propagator which can be separated in to two charged propagators; one in presence of a uniform background magnetic field $\left(S_{\ell}^{0}(p)\right)$ and the other in a magnetized medium $\left(S_{\ell}^{\beta}(p)\right)$ and can be written as

$$
S_{\ell}(p)=S_{\ell}^{0}(p)+S_{\ell}^{\beta}(p)
$$

Assuming that the z-axis points in the direction of the magnetic field B, we can express the charged lepton propagator in presence of a uniform background magnetic field as

$$
i S_{\ell}^{0}(p)=\int_{0}^{\infty} e^{\phi(p, s)} G(p, s) d s
$$


where the functions $\phi(p, s)$ and $G(p, s)$ are give by

$$
\begin{aligned}
\phi(p, s) & =i s\left(p_{0}^{2}-m_{\ell}^{2}\right)-i s\left[p_{3}^{2}+\frac{\tan z}{z} p_{\perp}^{2}\right], \\
G(p, s) & =\sec ^{2} z\left[\mathbf{A}+i \mathbf{B} \gamma_{5}+m_{\ell}\left(\cos ^{2} z-i \Sigma^{3} \sin z \cos z\right)\right],
\end{aligned}
$$

where $m_{l}$ is the mass of the charged lepton, $p_{\|}^{2}=p_{0}^{2}-p_{3}^{2}$ and $p_{\perp}^{2}=p_{1}^{2}+p_{2}^{2}$ are the projections of the momentum on the magnetic field direction and $z=e B s$, being $e$ the magnitude of the electron charge. Additionally, the covariant vectors are given as follows $A_{\mu}=p_{\mu}-\sin ^{2} z\left(p \cdot u u_{\mu}-p \cdot b b_{\mu}\right)$, $B_{\mu}=\sin z \cos z\left(p \cdot u b_{\mu}-p \cdot b u_{\mu}\right)$, and $\Sigma^{3}=\gamma_{5} b h$.

On the other hand, the charged lepton propagator in a magnetized medium is given by

$$
S_{\ell}^{\beta}(p)=i \eta_{F}(p \cdot u) \int_{-\infty}^{\infty} e^{\phi(p, s)} G(p, s) d s
$$

here $\eta_{F}(p \cdot u)$ contains the distribution functions of the particles in the medium which can be written as

$$
\eta_{F}(p \cdot u)=\frac{\theta(p \cdot u)}{e^{\beta\left(p \cdot u-\mu_{\ell}\right)}+1}+\frac{\theta(-p \cdot u)}{e^{-\beta\left(p \cdot u-\mu_{\ell}\right)}+1}
$$

where $\beta$ and $\mu_{\ell}$ are the inverse of the medium temperature and the chemical potential of the charged lepton, respectively.

The Z-exchange diagram for the one-loop self-energy is

$$
-i \Sigma_{Z}(k)=\mathcal{R}\left[\int \frac{d^{4} p}{(2 \pi)^{4}}\left(\frac{-i g}{\sqrt{2} \cos \theta_{W}}\right) \gamma_{\mu} i S_{\nu_{\ell}}(p)\left(\frac{-i g}{\sqrt{2} \cos \theta_{W}}\right) \gamma_{\nu} i Z^{\mu \nu}(q)\right] \mathcal{L}
$$

where $\theta_{W}$ is the Weinberg angle, $Z^{\mu \nu}(q)$ is the Z-boson propagator in vacuum, $S_{\nu_{l}}$ is the neutrino propagator in a thermal bath of neutrinos.

The Tadpole diagram for the one-loop self-energy is

$$
i \Sigma_{t}(k)=\mathcal{R}\left[\left(\frac{g}{2 \cos \theta_{W}}\right)^{2} \gamma_{\mu} i Z^{\mu \nu}(0) \int \frac{d^{4} p}{(2 \pi)^{4}} \operatorname{Tr}\left[\gamma_{\nu}\left(C_{V}+C_{A} \gamma_{5}\right) i S_{\ell}(p)\right]\right] \mathcal{L}
$$


where the quantities $C_{V}$ and $C_{A}$ are the vector and axial-vector coupling constants which come in the neutral-current interaction of electrons, protons $(p)$, neutrons $(n)$ and neutrinos with the $Z$ boson. Their forms are as follows

$$
C_{V}=\left\{\begin{array}{rc}
-\frac{1}{2}+2 \sin ^{2} \theta_{W} & e \\
\frac{1}{2} & \nu \\
\frac{1}{2}-2 \sin ^{2} \theta_{W} & p \\
-\frac{1}{2} & n
\end{array},\right.
$$

and

$$
C_{A}=\left\{\begin{array}{rc}
-\frac{1}{2} & \nu, p \\
\frac{1}{2} & e, n
\end{array} .\right.
$$

By evaluating eq. (8) explicitly we obtain

$$
\operatorname{Re} \Sigma_{W}(k)=\mathcal{R}\left[a_{W_{\perp}} k_{\perp}+b_{W} h+c_{W} b\right] \mathcal{L}
$$

where the Lorentz scalars are given by

$$
\begin{gathered}
a_{W \perp}=-\frac{\sqrt{2} G_{F}}{m_{W}^{2}}\left[\left\{E_{\nu_{e}}\left(n_{e}-\bar{n}_{e}\right)+k_{3}\left(n_{e}^{0}-\bar{n}_{e}^{0}\right)\right\}\right. \\
\left.+\frac{e B}{2 \pi^{2}} \int_{0}^{\infty} d p_{3} \sum_{n=0}^{\infty}\left(2-\delta_{n, 0}\right)\left(\frac{m_{e}^{2}}{E_{n}}-\frac{H}{E_{n}}\right)\left(f_{e, n}+\bar{f}_{e, n}\right)\right] \\
b_{W}=\sqrt{2} G_{F}\left[\left(1+\frac{3}{2} \frac{m_{e}^{2}}{m_{W}^{2}}+\frac{E_{\nu_{e}}^{2}}{m_{W}^{2}}\right)\left(n_{e}-\bar{n}_{e}\right)+\left(\frac{e B}{m_{W}^{2}}+\frac{E_{\nu_{e}} k_{3}}{m_{W}^{2}}\right)\left(n_{e}^{0}-\bar{n}_{e}^{0}\right)\right. \\
\left.-\frac{e B}{2 \pi^{2} m_{W}^{2}} \int_{0}^{\infty} d p_{3} \sum_{n=0}^{\infty}\left(2-\delta_{n, 0}\right)\left\{2 k_{3} E_{n} \delta_{n, 0}+2 E_{\nu_{e}}\left(E_{n}-\frac{m_{e}^{2}}{2 E_{n}}\right)\right\}\left(f_{e, n}+\bar{f}_{e, n}\right)\right](21)
\end{gathered}
$$

and

$$
\begin{aligned}
c_{W}= & \sqrt{2} G_{F}\left[\left(1+\frac{1}{2} \frac{m_{e}^{2}}{m_{W}^{2}}-\frac{k_{3}^{2}}{m_{W}^{2}}\right)\left(n_{e}^{0}-\bar{n}_{e}^{0}\right)+\left(\frac{e B}{m_{W}^{2}}-\frac{E_{\nu_{e}} k_{3}}{m_{W}^{2}}\right)\left(n_{e}-\bar{n}_{e}\right)\right. \\
& -\frac{e B}{2 \pi^{2} m_{W}^{2}} \int_{0}^{\infty} d p_{3} \sum_{n=0}^{\infty}\left(2-\delta_{n, 0}\right)\left\{2 E_{\nu_{e}}\left(E_{n}-\frac{m_{e}^{2}}{2 E_{n}}\right) \delta_{n, 0}\right.
\end{aligned}
$$




$$
\left.\left.+2 k_{3}\left(E_{n}-\frac{3}{2} \frac{m_{e}^{2}}{E_{n}}-\frac{H}{E_{n}}\right)\right\}\left(f_{e, n}+\bar{f}_{e, n}\right)\right]
$$

where the electron number density and electron distribution function are

$$
n_{e}(\mu, T, B)=\frac{e B}{2 \pi^{2}} \sum_{n=0}^{\infty}\left(2-\delta_{n, 0}\right) \int_{0}^{\infty} \frac{d p_{3}}{e^{\beta\left(E_{e, n}-\mu\right)}+1}
$$

and

$$
f\left(E_{e, n}, \mu\right)=\frac{1}{e^{\beta\left(E_{e, n}-\mu\right)}+1},
$$

respectively, with $\bar{f}_{e, n}(\mu, T)=f_{e, n}(-\mu, T)$ and $E_{e, n}=\sqrt{p_{3}^{2}+m_{e}^{2}+H}$ with $H=2 n e B$. We can also express the Eq. (15) (Z-exchange) as

$$
\operatorname{Re} \Sigma_{Z}(k)=\mathcal{R}\left(a_{Z} k+b_{Z} h\right) \mathcal{L}
$$

and explicit evaluation gives (D'Olivo \& Nieves 1994)

$$
a_{Z}=\sqrt{2} G_{F}\left[\frac{E_{\nu_{e}}}{m_{Z}^{2}}\left(n_{\nu_{e}}-\bar{n}_{\nu_{e}}\right)+\frac{2}{3} \frac{1}{m_{Z}^{2}}\left(\left\langle E_{\nu_{e}}\right\rangle n_{\nu_{e}}+\left\langle\bar{E}_{\nu_{e}}\right\rangle \bar{n}_{\nu_{e}}\right)\right]
$$

and

$$
b_{Z}=\sqrt{2} G_{F}\left[\left(n_{\nu_{e}}-\bar{n}_{\nu_{e}}\right)-\frac{8 E_{\nu}}{3 m_{Z}^{2}}\left(\left\langle E_{\nu_{e}}\right\rangle n_{\nu_{e}}+\left\langle\bar{E}_{\nu_{e}}\right\rangle \bar{n}_{\nu_{e}}\right)\right]
$$

where the four vector $k$ can be decomposed into the four vectors $h$ and $b$ in accordance with Eq. (3).

From the tadpole diagram, Eq. (16), we obtain

$$
\begin{aligned}
\operatorname{Re} \Sigma_{t}(k)= & \sqrt{2} G_{F} \mathcal{R}\left[\left\{C_{V_{e}}\left(n_{e}-\bar{n}_{e}\right)+C_{V_{p}}\left(n_{p}-\bar{n}_{p}\right)+C_{V_{n}}\left(n_{n}-\bar{n}_{n}\right)+\left(n_{\nu_{e}}-\bar{n}_{\nu_{e}}\right)\right.\right. \\
& \left.\left.+\left(n_{\nu_{\mu}}-\bar{n}_{\nu_{\mu}}\right)+\left(n_{\nu_{\tau}}-\bar{n}_{\nu_{\tau}}\right)\right\} u-C_{A_{e}}\left(n_{e}^{0}-\bar{n}_{e}^{0}\right) b\right] \mathcal{L} .
\end{aligned}
$$

For anti-neutrinos we must change $\left(\mathrm{n}_{x}-\bar{n}_{x}\right)$ by $-\left(\mathrm{n}_{x}-\bar{n}_{x}\right)$. The different contributions to the neutrino self-energy up to order the $1 / m_{W}^{4}$ have been calculated in a background of $\gamma, e^{ \pm}$, free baryons, neutrinos and anti-neutrinos. The effective potential that is applicable to neutrino oscillations in matter is $V_{\text {eff }}=V_{e}-V_{\mu, \tau}$ which depends only on electron density (Wolfenstein 1978; D'Olivo et 
al. 1992), assuming that neutrinos propagate in the same direction of the magnetic field $(\phi=0)$ and with $\mathrm{k}_{3}=\mathrm{E}_{\nu_{e}}$, we derive the neutrino effective potential in all the regimes of the magnetic field: strong $\Omega_{B}=e B / m_{e}^{2} \gg 1$, moderate $\Omega_{B}=e B / m_{e}^{2}>1$ (above $B_{c}$ ) and $\Omega_{B}=e B / m_{e}^{2} \leq 1$ (below $B_{c}$ ), and weak $\Omega_{B}=e B / m_{e}^{2} \ll 1$ regime, where $B_{c}=m_{e}^{2} / e=4.414 \times 10^{13} \mathrm{G}$ is the critical magnetic field. In the following subsections we will show the neutrino effective potential in all the regimes (strong, moderate and weak magnetic field limit)(Erdas 2009); the calculations are explicitly shown in the appendix. Using the typical fireball values at the initial stage and the phase of acceleration; temperature in the range $1-10 \mathrm{MeV}$ at initial radius $\mathrm{r}_{0} \simeq 10^{6.5}-10^{7.5} \mathrm{~cm}$ for thermal MeV neutrinos (Beloborodov 2003a; Ruffert \& Janka 1999; Koers \& Wijers 2005, Janka et al. 2012) and in the range $0.1-1 \mathrm{MeV}$ at radius $\mathrm{r} \simeq 10^{8.5}-10^{9.5} \mathrm{~cm}$ for quasi-thermal GeV neutrinos (Bahcall \& meszaros 2000; Meszaros \& Rees 2000; Razzaque \& Meszaros 2006a b; Murase et al. 2013), we plot the effective potential in each limit as shown in figs. 2, 3, 4 and 5. Although we are going to do a full analysis of neutrino oscillations in a fireball endowed with moderate magnetic fields which is more favored for GRB central engines (Beloborodov 2003a; Mészáros 2012), a brief and additional description at the strong and weak field limit as well as a comparison of effective potentials at all the regime will be given as follows.

\subsection{Strong Magnetic field: $\Omega_{B} \gg 1$}

In the strong magnetic field approximation $\left(m_{e}^{2} \ll T^{2} \ll \Omega_{B} m_{e}^{2}\right)$, leptons are all confined to the lowest Landau level $(n=0)$, then only this level will contribute to the potential and the energy of these leptons will be independent of the magnetic field. The neutrino effective potential at the strong magnetic field is given by

$V_{e f f, S}=A_{e}\left[\sum_{l=0}^{\infty}(-1)^{l} \sinh \alpha_{l} K_{1}\left(\sigma_{l}\right)\left\{\frac{m_{e}^{2}}{m_{W}^{2}}\left(1+4 \frac{E_{\nu}^{2}}{m_{e}^{2}}\right)\right\}-3 \frac{m_{e}^{2}}{m_{W}^{2}} \frac{E_{\nu}}{m_{e}} \sum_{l=0}^{\infty}(-1)^{l} \cosh \alpha_{l} K_{0}\left(\sigma_{l}\right)\right]$. 
In fig. 2, we plot the effective potential at the strong field limit (eq. 29) as a function of temperature (left-hand figure above), magnetic field (right-hand figure above) and chemical potential (figure below). For these plots we take into account the neutrino energy $10 \mathrm{MeV}$ and the values of temperature, magnetic field and chemical potential in the range 1 to $10 \mathrm{MeV}, 10^{3}$ to $10^{5} B_{c}$ and $10^{-4} \mathrm{eV}$ to $4.5 \mathrm{keV}$, respectively. As shown, the effective potential is a quasi-constant function of temperature, and an increasing function of magnetic field and chemical potential

\subsection{Moderate Magnetic field: $\Omega_{B}>1$ and $\Omega_{B} \leq 1$}

In the moderate field approximation $\left(m_{e}^{2}<\Omega_{B} m_{e}^{2} \leq T^{2}\right)$, leptons start to occupy the next Landau levels $(\mathrm{n}=1,2,3 .$.$) which have a separation that is directly proportional to magnetic$ field. For this case each of these levels will contribute to the effective potential and the energy of leptons are directly proportional to magnetic field. The neutrino effective potential in the moderate magnetic field is written as

$$
\begin{gathered}
V_{e f f}=A_{e}\left[\sum_{l=0}^{\infty}(-1)^{l} \sinh \alpha_{l}\left\{\frac{m_{e}^{2}}{m_{W}^{2}}\left(1+4 \frac{E_{\nu}^{2}}{m_{e}^{2}}\right) K_{1}\left(\sigma_{l}\right)+\sum_{n=1}^{\infty} \lambda_{n}\left(2+\frac{m_{e}^{2}}{m_{W}^{2}}\left(3-2 \Omega_{B}+4 \frac{E_{\nu}^{2}}{m_{e}^{2}}\right)\right) K_{1}\left(\sigma_{l} \lambda_{n}\right)\right\}\right. \\
\left.-4 \frac{m_{e}^{2}}{m_{W}^{2}} \frac{E_{\nu}}{m_{e}} \sum_{l=0}^{\infty}(-1)^{l} \cosh \alpha_{l}\left\{\frac{3}{4} K_{0}\left(\sigma_{l}\right)+\sum_{n=1}^{\infty} \lambda_{n}^{2} K_{0}\left(\sigma_{l} \lambda_{n}\right)\right\}\right]
\end{gathered}
$$

with

$$
\lambda^{2}= \begin{cases}2 n \Omega_{B} & \text { for } \Omega_{B}>1 \\ 1+2 n \Omega_{B} & \text { for } \Omega_{B} \leq 1:\end{cases}
$$

In fig. 3, we have plotted the neutrino effective potential at the moderate field limit above $B_{c}$ (eq. 30) as a function of temperature (left-hand figure above), magnetic field (right-hand figure above) and chemical potential (figure below). For these plots we take into account the neutrino energy 10 $\mathrm{MeV}$ and the values of temperature, magnetic field and chemical potential in the range 1 to 10 $\mathrm{MeV}, B_{c}<B \leq 10^{2} B_{c}$ and $10^{-4} \mathrm{eV}$ to $4.5 \mathrm{keV}$, respectively. As shown, the effective potential as a function of temperature has two different behaviors. Firstly, it is an increasing function of temperature in the range 1 to $3 \mathrm{MeV}$ for values of magnetic field $5 B_{c}$ and $10 B_{c}$, and in the range 
1 to $7 \mathrm{MeV}$ for $50 B_{c}$ and $100 B_{c}$ and secondly, it tends to be constant for values of temperature greater than $3 \mathrm{MeV}$ for $B=5 B_{c}$ and $B=10 B_{c}$, and $7 \mathrm{MeV}$ for $B=50 B_{c}$ and $B=100 B_{c}$. In this figure it is also seen that the effective potential is an increasing function for both the magnetic field and chemical potential.

In fig. 4, we have plotted the neutrino effective potential at the moderate field limit below $\mathrm{B}_{c}$ (eq. 30) as a function of temperature (figure above), magnetic field (middle figure) and chemical potential (figure below) for neutrino energies $E_{\nu}=10$ (left column figures) $\mathrm{MeV}$ and $E_{\nu}=10$ $\mathrm{GeV}$ (left column figures). For these plots we take into account the values of the magnetic field and chemical potential in the range $10^{-3} B_{c}<B \leq B_{c}$ and $10^{-4} \mathrm{eV}$ to $4.5 \mathrm{keV}$, respectively, and

two ranges of temperatures; $0.1 \mathrm{MeV} \leq T \leq 1 \mathrm{MeV}$ (right column figures) and $1 \mathrm{MeV} \leq T \leq 10$ $\mathrm{MeV}$ (left column figures). In the top figures, the behavior of the effective potential as a function of temperature has a multifunctional dependence which depends on the values of the magnetic field. For instance, in the right-hand figure, for $\mathrm{B}=0.5 \mathrm{~B}_{c}$ it is a dramatically increasing function, for $\mathrm{B}=10^{-2} B_{c}$ it is just a steadily increasing function and for the smaller values of $\mathrm{B}$, it becomes a decreasing function and in the left-hand figure, for $\mathrm{B}=0.5 B_{c}$ it is a steadily increasing function and as B decreases the effective potential gradually becomes a decreasing function. In the middle figures, the effective potential is a dramatically (left-hand figure) and (right-hand figure) steadily increasing function of the magnetic field regardless of the values of temperature. In the bottom figures, the effective potential represented by means of an increasing function of the chemical potential shows the same behavior but at different energy ranges.

\subsection{Weak Magnetic field: $\Omega_{B} \ll 1$}

In the weak field approximation $\left(\Omega_{B} m_{e}^{2} \ll m_{e}^{2}\right)$, all Landau levels are occupied and overlapped each other, therefore a good description of these levels is to take the approximation 
$\sum_{n} \rightarrow \int d n$. For this case, the neutrino effective potential is

$$
\begin{aligned}
V_{e f f, W}=A_{e} & {\left[\sum_{l=0}^{\infty}(-1)^{l} \sinh \alpha_{l}\left\{\left(2+\frac{m_{e}^{2}}{m_{W}^{2}}\left(3+4 \frac{E_{\nu}^{2}}{m_{e}^{2}}\right)\right)\left(\frac{K_{0}\left(\sigma_{l}\right)}{\sigma_{l}}+2 \frac{K_{1}\left(\sigma_{l}\right)}{\sigma_{l}^{2}}\right) \Omega_{B}^{-1}-2\left(1+\frac{m_{e}^{2}}{m_{W}^{2}}\right) K_{1}\left(\sigma_{l}\right)\right\}\right.} \\
& \left.-4 \frac{m_{e}^{2}}{m_{W}^{2}} \frac{E_{\nu}}{m_{e}} \sum_{l=0}^{\infty}(-1)^{l} \cosh \alpha_{l}\left\{\left(\frac{2}{\sigma_{l}^{2} \Omega_{B}}-\frac{1}{4}\right) K_{0}\left(\sigma_{l}\right)+\left(1+\frac{4}{\sigma_{l}^{2}}\right) \frac{K_{1}\left(\sigma_{l}\right)}{\sigma_{l}} \Omega_{B}^{-1}\right\}\right] .
\end{aligned}
$$

As shown in fig. 5 the neutrino effective potential at the weak field limit (eq. 31) as a function of temperature (figures above) and chemical potential (figures below) for neutrino energies $\mathrm{E}_{\nu}=10$ $\mathrm{MeV}$ (left-hand figures) and $\mathrm{E}_{\nu}=10 \mathrm{GeV}$ (left-hand figures) is plotted. Due to the strength of the magnetic field it is quite small and any variation will produce insignificant changes in the effective potential; we only show the magnetic field contribution, i.e. by subtracting the effective potential with $\mathrm{B}=0$, which shows that the potential is a decreasing function of temperature and an increasing function of chemical potential, and that the magnetic contribution is the opposite as compared with the medium contribution. The effective potential in this regime differs from that calculated by Sahu et al. (2009b), because the authors took the solution of dispersion relation $k_{3}=-E_{\nu_{e}}$ instead of $k_{3}=E_{\nu_{e}}$.

\subsection{Comparison of effective potentials at all the regimes}

We plot the neutrino effective potentials as a function of temperature (left-hand figure) and chemical potential (right-hand figure) in a strong, moderate and weak magnetic field approximation (fig. 6) which are given in eqs. (29), (30) and (31), respectively. As shown in fig. 6. the effective potential lies between $\sim 10^{-11}$ and $\sim 10^{-8} \mathrm{eV}$ for a temperature in the range 1 to $8 \mathrm{MeV}$. One can see from this plot several features. i) at $\mathrm{T} \sim 1 \mathrm{MeV}$, the effective potentials at moderate (above and below $B_{c}$ ) field limit are quite close at $\sim 5 \times 10^{-10} \mathrm{eV}$, however as temperature increases the separation between them also increases and for strong and weak field limit, the distance between the effective potentials is more than two orders of magnitude. ii) As 
temperature increases the effective potentials at the strong and moderate (above $B_{c}$ ) field limits become closer to each other. Dividing this plot in two regions, $\mathrm{T}<3 \mathrm{MeV}$ and $\mathrm{T} \geq 3 \mathrm{MeV}$, we can argue that for the given values of the magnetic field and temperature, in the first region $\mathrm{m}_{e}^{2} \Omega_{B} \simeq T^{2}$, even though being slightly lower at $\mathrm{T} \sim 1.4 \mathrm{MeV}$ and as temperature becomes larger than the magnetic field, $\mathrm{m}_{e}^{2} \Omega_{B} \ll T^{2}$, a difference between both effective potentials for each value of temperature is observed. This difference, which is of the same order of magnitude, comes from the contribution of excited Landau levels ( $n=1,2$..). iii) The effective potential at the weak field limit is a dramatically increasing function of temperature which tends at the moderate limit below $B_{c}$. iv) The effective potential at strong field limit decreases very gradually (quasi constantly) as temperature increases, in fact for the range of temperature considered, the effective potential is almost invariant to any thermal contribution. Also it is an increasing function of the chemical potential in the range $0.1-4 \times 10^{3} \mathrm{eV}$ for all the regimes of the magnetic fields. By comparison the effective potential at strong (maximum value) and weak (minimum value) field approximation, one can see that the last approximation is smaller by three orders of magnitude than the first one. Additionally, we plot the contribution of $\mathrm{m}_{W}^{-4}$ terms to the neutrino effective potential as shown in fig. 7. As can be seen in eqs. (29), 30) and (31), there are two common terms which depend on $\mathrm{m}_{W}^{-4}$ and that contribute to the effective potentials ; $(-1)^{l} \cosh (\alpha) K_{0}(\sigma)$ and $4 E_{\nu}^{2} / m_{W}^{2}$. In the first case, we compare the terms $(-1)^{l} \sinh (\alpha) K_{1}(\sigma)$ and $(-1)^{l} \cosh (\alpha) K_{0}(\sigma)$ (left-hand figure) where the first term comes from particle-antiparticle asymmetry $\left(n_{e}-\bar{n}_{e}\right)$ and the second one from $\left(n_{e}+\bar{n}_{e}\right)$. From this figure, one can observe that the $\left(n_{e}-\bar{n}_{e}\right) /\left(n_{e}+\bar{n}_{e}\right)$ ratio is directly proportional to chemical potential and for $\mu \leq 10^{2} \mathrm{eV}$ the $\mathrm{m}_{W}^{-4}$ term begins to be dominant achieving a minimum value of 7 orders of magnitude for $\mu=10^{-3} \mathrm{eV}$. In other words, as $\mu$ decreases the correction of order $\mathrm{m}_{W}^{-4}$ is important and dominant over $\mathrm{m}_{W}^{-2}$ term, even though one can deduce that for $\mu=0, n_{e}-\bar{n}_{e}=0$, then the only contribution would come from the term $\mathrm{O}\left(\mathrm{m}_{W}^{-4}\right)$. In the second case we plot $4 E_{\nu}^{2} / m_{W}^{2}$ as a function of $\mathrm{E}_{\nu}$ (right-hand figure). From this plot, you can notice that this term in comparison with unity starts to contribute for neutrinos with 
energies of some tens of GeV. As shown above, these two terms with the values of temperature, chemical potential and neutrino energy are relevant to the neutrino effective potential.

From the previous analysis can be observed that regardless of the magnetic field limit or relativistic temperature or chemical potential, the neutrino effective potential is positive, therefore due to its positivity $\left(V_{e f f, k}>0\right.$ for $\mathrm{k}=\mathrm{S}, \mathrm{M}$ and $\left.\mathrm{W}\right), \mathrm{MeV}-\mathrm{GeV}$ neutrinos can oscillate resonantly.

\section{Neutrino Mixing and Resonance Condition}

In the following subsections we are going to consider the best fit values of the neutrino oscillation parameters for two-neutrino mixing (solar, atmospheric and accelerator) and three-neutrino mixing.

\subsection{Two-Neutrino Mixing}

Here we consider the neutrino oscillation process $\nu_{e} \leftrightarrow \nu_{\mu, \tau}$. The evolution equation for the propagation of neutrinos in the medium is given by (Fraija 2014a)

$$
i\left(\begin{array}{c}
\dot{\nu}_{e} \\
\dot{\nu}_{\mu}
\end{array}\right)=\left(\begin{array}{cc}
V_{e f f}-\Delta \cos 2 \theta & \frac{\Delta}{2} \sin 2 \theta \\
\frac{\Delta}{2} \sin 2 \theta & 0
\end{array}\right)\left(\begin{array}{c}
\nu_{e} \\
\nu_{\mu}
\end{array}\right),
$$

where $\Delta=\delta m^{2} / 2 E_{\nu}, \delta m^{2}$ is the mass difference, $V_{e f f}$ is the neutrino effective potential between $V_{\nu_{e}}$ and $V_{\nu_{\mu, \tau}}$ (eq. 30), $E_{\nu}$ is the neutrino energy and $\theta$ is the neutrino mixing angle. The conversion probability for the above process at a time $t$ is given by

$$
P_{\nu_{e} \rightarrow \nu_{\mu}\left(\nu_{\tau}\right)}(t)=\frac{\Delta^{2} \sin ^{2} 2 \theta}{\omega^{2}} \sin ^{2}\left(\frac{\omega t}{2}\right)
$$

with

$$
\omega=\sqrt{\left(V_{e f f}-\Delta \cos 2 \theta\right)^{2}+\Delta^{2} \sin ^{2} 2 \theta}
$$


The oscillation length for the neutrino is given by

$$
l_{\text {osc }}=\frac{l_{v}}{\sqrt{\cos ^{2} 2 \theta\left(1-\frac{V_{e f f}}{\Delta \cos 2 \theta}\right)^{2}+\sin ^{2} 2 \theta}},
$$

where $l_{v}=2 \pi / \Delta$ is the vacuum oscillation length. Applying the resonance condition given by

$$
\begin{aligned}
V_{\text {eff }} & =\Delta \cos 2 \theta \\
& =5 \times 10^{-7} \mathrm{eV} \frac{\delta m_{e V}^{2}}{E_{\nu, M e V}} \cos 2 \theta
\end{aligned}
$$

we obtain that the resonance length $\left(l_{\text {res }}\right)$ can be written as

$$
l_{\text {res }}=\frac{l_{v}}{\sin 2 \theta}
$$

In eq. (36) the neutrino effective potential depends on the chemical potential, temperature, the neutrino energy and the oscillation parameters (mass differences and the mixing angles). We will use the following parameters for this analysis:

Solar Neutrinos: A two-flavor neutrino oscillation analysis yielded $\delta m^{2}=\left(5.6_{-1.4}^{+1.9}\right) \times 10^{-5} \mathrm{eV}^{2}$ and $\tan ^{2} \theta=0.427_{-0.029}^{+0.033}$ Aharmin et al. 2011).

Atmospheric Neutrinos: Under a two-flavor disappearance model with separate mixing parameters between neutrinos and antineutrinos the following parameters for the SK-I + II + III data $\delta m^{2}=\left(2.1_{-0.4}^{+0.9}\right) \times 10^{-3} \mathrm{eV}^{2}$ and $\sin ^{2} 2 \theta=1.0_{-0.07}^{+0.00}$ were found.(Abe et al. 2011a). Accelerator Parameters (Short baselines): Church et al. (2002) found two well defined regions of oscillation parameters with either $\delta m^{2} \approx 7 \mathrm{eV}^{2}$ or $\delta m^{2}<1 \mathrm{eV}^{2}$ compatible with both LAND and KARMEN experiments, for the complementary confidence. In addition, MiniBooNE found evidence of oscillations in the 0.1 to $1.0 \mathrm{eV}^{2}$, which are consistent with LSND results (Athanassopoulos et al. 1996, 1998). 


\subsection{Three-Neutrino Mixing}

To determine the neutrino oscillation probabilities we have to solve the evolution equation of the neutrino system in the matter. In a three-flavor framework, this equation is given by

$$
i \frac{d \vec{\nu}}{d t}=H \vec{\nu}
$$

and the state vector in the flavor basis is defined as

$$
\vec{\nu} \equiv\left(\nu_{e}, \nu_{\mu}, \nu_{\tau}\right)^{T}
$$

The effective Hamiltonian is

$$
H=U \cdot H_{0}^{d} \cdot U^{\dagger}+\operatorname{diag}\left(V_{e f f}, 0,0\right),
$$

with

$$
H_{0}^{d}=\frac{1}{2 E_{\nu}} \operatorname{diag}\left(-\delta m_{21}^{2}, 0, \delta m_{32}^{2}\right) .
$$

Here $V_{\text {eff }}$ is the effective potential (eq. 30) and $U$ is the three neutrino mixing matrix given by (Gonzalez-Garcia 2003; Akhmedov et al. 2004; Gonzalez-Garcia \& Maltoni 2008; Gonzalez-Garcia 2011),

$$
U=\left(\begin{array}{ccc}
c_{13} c_{12} & s_{12} c_{13} & s_{13} \\
-s_{12} c_{23}-s_{23} s_{13} c_{12} & c_{23} c_{12}-s_{23} s_{13} s_{12} & s_{23} c_{13} \\
s_{23} s_{12}-s_{13} c_{23} c_{12} & -s_{23} c_{12}-s_{13} s_{12} c_{23} & c_{23} c_{13}
\end{array}\right)
$$

where $s_{i j}=\sin \theta_{i j}$ and $c_{i j}=\cos \theta_{i j}$. For anti-neutrinos one has to replace $U$ by $U^{*}$. The different neutrino probabilities are given as

$$
\begin{aligned}
P_{e e} & =1-4 s_{13, m}^{2} c_{13, m}^{2} S_{31}, \\
P_{\mu \mu} & =1-4 s_{13, m}^{2} c_{13, m}^{2} s_{23}^{4} S_{31}-4 s_{13, m}^{2} s_{23}^{2} c_{23}^{2} S_{21}-4 c_{13, m}^{2} s_{23}^{2} c_{23}^{2} S_{32}, \\
P_{\tau \tau} & =1-4 s_{13, m}^{2} c_{13, m}^{2} c_{23}^{4} S_{31}-4 s_{13, m}^{2} s_{23}^{2} c_{23}^{2} S_{21}-4 c_{13, m}^{2} s_{23}^{2} c_{23}^{2} S_{32}, \\
P_{e \mu} & =4 s_{13, m}^{2} c_{13, m}^{2} s_{23}^{2} S_{31},
\end{aligned}
$$




$$
\begin{aligned}
& P_{e \tau}=4 s_{13, m}^{2} c_{13, m}^{2} c_{23}^{2} S_{31} \\
& P_{\mu \tau}=-4 s_{13, m}^{2} c_{13, m}^{2} s_{23}^{2} c_{23}^{2} S_{31}+4 s_{13, m}^{2} s_{23}^{2} c_{23}^{2} S_{21}+4 c_{13, m}^{2} s_{23}^{2} c_{23}^{2} S_{32}
\end{aligned}
$$

where

$$
\sin 2 \theta_{13, m}=\frac{\sin 2 \theta_{13}}{\sqrt{\left(\cos 2 \theta_{13}-2 E_{\nu} V_{e f f} / \delta m_{32}^{2}\right)^{2}+\left(\sin 2 \theta_{13}\right)^{2}}}
$$

and

$$
S_{i j}=\sin ^{2}\left(\frac{\Delta \mu_{i j}^{2}}{4 E_{\nu}} l_{o s c}\right)
$$

with

$$
\begin{aligned}
\Delta \mu_{21}^{2} & =\frac{\delta m_{32}^{2}}{2}\left(\frac{\sin 2 \theta_{13}}{\sin 2 \theta_{13, m}}-1\right)-E_{\nu} V_{\text {eff }} \\
\Delta \mu_{32}^{2} & =\frac{\delta m_{32}^{2}}{2}\left(\frac{\sin 2 \theta_{13}}{\sin 2 \theta_{13, m}}+1\right)+E_{\nu} V_{\text {eff }} \\
\Delta \mu_{31}^{2} & =\delta m_{32}^{2} \frac{\sin 2 \theta_{13}}{\sin 2 \theta_{13, m}},
\end{aligned}
$$

and

$$
\begin{aligned}
\sin ^{2} \theta_{13, m} & =\frac{1}{2}\left(1-\sqrt{1-\sin ^{2} 2 \theta_{13, m}}\right) \\
\cos ^{2} \theta_{13, m} & =\frac{1}{2}\left(1+\sqrt{1-\sin ^{2} 2 \theta_{13, m}}\right)
\end{aligned}
$$

The oscillation length for the neutrino is given by

$$
l_{o s c}=\frac{l_{v}}{\sqrt{\cos ^{2} 2 \theta_{13}\left(1-\frac{2 E_{\nu} V_{e}}{\delta m_{32}^{2} \cos 2 \theta_{13}}\right)^{2}+\sin ^{2} 2 \theta_{13}}},
$$

where $l_{v}=4 \pi E_{\nu} / \delta m_{32}^{2}$ is the vacuum oscillation length. The resonance condition and resonance length are,

$$
V_{e f f}-5 \times 10^{-7} \frac{\delta m_{32, e V}^{2}}{E_{\nu, M e V}} \cos 2 \theta_{13}=0
$$

and

$$
l_{r e s}=\frac{l_{v}}{\sin 2 \theta_{13}}
$$


Considering the adiabatic condition at the resonance, we can express it as,

$$
\begin{aligned}
\kappa_{\text {res }} & \equiv \frac{2}{\pi}\left(\frac{\delta m_{32}^{2}}{2 E_{\nu}} \sin 2 \theta_{13}\right)^{2}\left(\frac{d V_{\text {eff }}}{d r}\right)^{-1} \geq 1 \\
& =3.62 \times 10^{-2} \mathrm{~cm}^{-1}\left(\frac{\delta m_{32, \mathrm{eV}}^{2}}{E_{\nu, M e V}} \sin 2 \theta_{13}\right)^{2} \frac{1}{V^{\prime}} \geq 1
\end{aligned}
$$

where

$$
V^{\prime}=\Omega_{B}\left[\frac{d V_{e f f, 1}}{d r}-3.16 \times 10^{-10} E_{\nu, M e V} \frac{d V_{e f f, 2}}{d r}\right]
$$

and

$$
\begin{aligned}
V_{e f f, 1}= & \sum_{l=0}^{\infty}(-1)^{l} \sin \alpha_{l}\left\{\frac{m_{e}^{2}}{m_{W}^{2}}\left(1+4 \frac{E_{\nu}^{2}}{m_{e}^{2}}\right) K_{1}\left(\sigma_{l}\right)\right. \\
& \left.+\sum_{n=1}^{\infty} \lambda_{n}\left[2+\frac{m_{e}^{2}}{m_{W}^{2}}\left(3+4 \frac{E_{\nu}^{2}}{m_{e}^{2}}-2 \Omega_{B}\right)\right] K_{1}\left(\sigma_{l} \lambda_{n}\right)\right\} \\
V_{e f f, 2}= & \sum_{l=0}^{\infty}(-1)^{l} \cos \alpha_{l}\left(\frac{3}{4} K_{0}\left(\sigma_{l}\right)+\sum_{n=1}^{\infty} \lambda_{n}^{2} K_{0}\left(\sigma_{l} \lambda_{n}\right)\right)
\end{aligned}
$$

We will use the following parameters (Aharmin et al. 2011; Wendell et al. 2010) for this analysis:

$$
\begin{aligned}
& \text { for } \sin _{13}^{2}<0.053: \delta m_{21}^{2}=\left(7.41_{-0.19}^{+0.21}\right) \times 10^{-5} \mathrm{eV}^{2} \text { and } \tan ^{2} \theta_{12}=0.446_{-0.029}^{+0.030} \\
& \text { for } \sin _{13}^{2}<0.04: \delta m_{23}^{2}=\left(2.1_{-0.2}^{+0.5}\right) \times 10^{-3} \mathrm{eV}^{2} \text { and } \sin ^{2} \theta_{23}=0.50_{-0.093}^{+0.083}
\end{aligned}
$$

For a complete description of resonant neutrino oscillations at early fireball stage, we use the values of fireball observables during the initial stage and phase of acceleration. At the initial stage, we consider a fireball endowed with magnetic field $B=10 B_{c}$, temperature in the range 1 to $5 \mathrm{MeV}$ and thermal neutrino energies $\mathrm{E}_{\nu}=1,5,20$ and $30 \mathrm{MeV}$ whereas at the acceleration phase the corresponding values considered are: magnetic field $B=10^{-4.3} B_{c}$, temperature in the range 50 to $500 \mathrm{keV}$ and quasi-thermal $\mathrm{GeV}$ neutrino energies $\mathrm{E}_{\nu}=1,10,20$ and $50 \mathrm{GeV}$. In both cases we take the best fit of parameters for two- (solar: $\delta m^{2}=5.6 \times 10^{-5} \mathrm{eV}^{2}$ and $\tan ^{2} \theta=0.427$ (Aharmin et al. 2011), atmospheric: $\delta m^{2}=2.1 \times 10^{-3} \mathrm{eV}^{2}$ and $\sin ^{2} 2 \theta=1.0$ (Abe et al. 2011a), and accelerator: $\delta m^{2} \sim 0.6 \mathrm{eV}^{2}$ and $\sin ^{2} 2 \theta=51.2 \times 10^{-3}$ (Church et al. 2002) ) and three $\left(\delta m_{32}^{2}=10^{-2.58}\right.$ and $\left.\theta_{13}=11^{\circ}\right)$-neutrino mixing in order to analyze the resonance conditions in 
both phases.

From the resonance conditions (eqs. 36 and 49), we have obtained the contour plots of temperature and chemical potential at the initial stage (fig. 8) and phase of acceleration (fig. 9). At the initial phase, chemical potential lies in the range $7.4 \times 10^{-3}$ to $3.01 \mathrm{eV}$ for solar parameters, $1.01 \times 10^{-2}$ to $2.84 \mathrm{eV}$ for atmospheric parameters, 0.12 to $3.1 \times 10^{2} \mathrm{keV}$ for accelerator parameters and 0.8 to $50 \times 10^{2} \mathrm{eV}$ for three-neutrino mixing. One can see that temperature is a decreasing function of chemical potential which gradually increases as neutrino energy is decreased. In addition, we have computed the resonance lengths which are shown in table 1. As shown in this table, the resonance lengths lie in the range $l_{\text {res }} \sim\left(10^{4}\right.$ to $\left.10^{8}\right) \mathrm{cm}$ which are comparable with the length scale of a fireball. Therefore, depending on the oscillation parameters neutrinos could oscillate resonantly before leaving the fireball. For instance, considering an initial radius of $10 \mathrm{~km}$, only neutrinos with low energy will oscillate resonantly for atmospheric, accelerator and three-neutrino mixing but no solar parameters. If we assume an initial radius of $100 \mathrm{~km}$, and consider atmospheric and accelerator oscillation parameters, then neutrinos would oscillate resonantly regardless their energies. Once again, assuming a radius of $1000 \mathrm{~km}$, thermal neutrinos will oscillate resonantly. In the phase of acceleration, chemical potential lies in the range $2.2 \times 10^{-2}$ to $1 \mathrm{eV}$ for solar, $4.01 \times 10^{-2}$ to $1.3 \mathrm{eV}$ for atmospheric, $1.1 \times 10^{-2}$ to $0.5 \mathrm{keV}$ for accelerator and 0.5 to $6.1 \mathrm{eV}$ for three-neutrino mixing. One can see that temperature is a decreasing function of chemical potential which increases as neutrino energy increases (decreases) for solar and atmospheric (accelerator) oscillation parameters and it is doubly degenerate for three-neutrino mixing. Further, we have computed the resonance lengths of $\mathrm{GeV}$ neutrinos. As shown in table 2, the resonance lengths match the length scale where they were created for three-neutrino mixing. In other words, taking into account the former parameters, multi GeV neutrinos created at $\sim\left(10^{11}-10^{13}\right) \mathrm{cm}$ will have simultaneously resonant oscillations. Finally, we have studied the survival and conversion probability for the active-active $\left(\nu_{e, \mu, \tau} \leftrightarrow \nu_{e, \mu, \tau}\right)$ neutrino oscillations and the three-neutrino mixing. In fig. 10 the survival probability of electron $P_{e e}$, muon $P_{\mu \mu}$ and tau $P_{\tau \tau}$ neutrino and 


\begin{tabular}{ccccc}
\hline $\begin{array}{c}\text { Energy } \\
(\mathrm{MeV})\end{array}$ & Solar & Atmosph. & Accelerat. & Three flavors \\
\hline 1 & $4.8 \times 10^{6}$ & $1.2 \times 10^{5}$ & $7.1 \times 10^{3}$ & $2.5 \times 10^{5}$ \\
5 & $2.4 \times 10^{7}$ & $5.9 \times 10^{5}$ & $3.6 \times 10^{4}$ & $1.3 \times 10^{6}$ \\
20 & $1.0 \times 10^{8}$ & $2.4 \times 10^{6}$ & $1.4 \times 10^{5}$ & $5.0 \times 10^{7}$ \\
30 & $1.5 \times 10^{8}$ & $3.5 \times 10^{6}$ & $2.1 \times 10^{5}$ & $7.6 \times 10^{7}$ \\
\hline
\end{tabular}

Table 1: Resonance lengths of thermal neutrinos for the best fit parameters of two- and threeneutrino mixing.

\begin{tabular}{ccrlc}
\hline $\begin{array}{c}\text { Energy } \\
(\mathrm{GeV})\end{array}$ & Solar & Atmosph. & Accelerat. & Three flavors \\
\hline 5 & $2.4 \times 10^{10}$ & $5.9 \times 10^{8}$ & $3.6 \times 10^{7}$ & $1.3 \times 10^{9}$ \\
10 & $4.8 \times 10^{10}$ & $1.2 \times 10^{9}$ & $7.1 \times 10^{7}$ & $2.5 \times 10^{9}$ \\
20 & $9.6 \times 10^{10}$ & $2.4 \times 10^{9}$ & $1.4 \times 10^{8}$ & $5.0 \times 10^{9}$ \\
50 & $2.4 \times 10^{11}$ & $5.9 \times 10^{9}$ & $3.6 \times 10^{8}$ & $1.3 \times 10^{10}$ \\
\hline
\end{tabular}

Table 2: Resonance lengths of quasi-thermal neutrinos for the best fit parameters of the two- and three-neutrino mixing. 
conversion probabilities $P_{e \mu}, P_{e \tau}$ and $P_{\mu \tau}$ as a function of energy for fixed values of length scale, temperature, magnetic field and neutrino energy is plotted. In the left-hand figures, we use once again the fireball values in the initial stage: $\mathrm{T}=5 \mathrm{MeV}, \mathrm{r}_{0}=100 \mathrm{~km}$ (above) and $r_{0}=10 \mathrm{~km}$ (below), $\mathrm{B}=10 B_{c}$, neutrino energy range 1 to $30 \mathrm{MeV}$ and in the right-hand figures we take into account the values already mentioned for the phase of acceleration: $\mathrm{T}=100 \mathrm{keV}, \mathrm{r}=10^{11} \mathrm{~cm}$ (above) and $\mathrm{r}=10^{12} \mathrm{~cm}$ (below), $\mathrm{B}=10^{-4.3} B_{c}$, neutrino energy range $1-30 \mathrm{GeV}$. Our analysis shows that $P_{e e}=1, P_{e \mu}=P_{e \tau}=0$, indicating that the propagating electron neutrinos do not oscillate to any other flavor independently of the their energies and radii. Instead, it tells us that muon and tau neutrinos oscillate among themselves with equal probability and that the oscillation depends on their energy and distances. As can be seen, the probabilities satisfy the condition

$$
\sum_{i=e, \mu, \tau} P_{e i}\left(\delta m_{32}^{2}, L\right)=1, \quad \sum_{i=e, \mu, \tau} P_{\mu i}\left(\delta m_{32}^{2}, L\right)=1, \quad \sum_{i=e, \mu, \tau} P_{\tau i}\left(\delta m_{32}^{2}, L\right)=1 .
$$

\subsection{Neutrino Oscillation from Source to Earth}

Between the surface of the star and the Earth the flavor ratio $\phi_{\nu_{e}}^{0}: \phi_{\nu_{\mu}}^{0}: \phi_{\nu_{\tau}}^{0}$ is affected by the full three description flavor mixing, which is calculated as follows. The probability for a neutrino to oscillate from a flavor state $\alpha$ to a flavor state $\beta$ in a time starting from the emission of neutrino at $\operatorname{star} \mathrm{t}=0$, is given as

$$
\begin{aligned}
P_{\nu_{\alpha} \rightarrow \nu_{\beta}} & =\left|<\nu_{\beta}(t)\right| \nu_{\alpha}(t=0)>\mid \\
& =\delta_{\alpha \beta}-4 \sum_{j>i} U_{\alpha i} U_{\beta i} U_{\alpha j} U_{\beta i} \sin ^{2}\left(\frac{\delta m_{i j}^{2} l_{o s c}}{4 E_{\nu}}\right) .
\end{aligned}
$$

Using the set of parameters given in eq. (54), we can write the mixing matrix

$$
U=\left(\begin{array}{ccc}
0.816669 & 0.544650 & 0.190809 \\
-0.504583 & 0.513419 & 0.694115 \\
0.280085 & -0.663141 & 0.694115
\end{array}\right)
$$

Additionally, averaging the sin term in the probability to $\sim 0.5$ for larger distances $l_{\text {osc }}$ (Learned \& Pakvasa 1995), the probability matrix for a neutrino flavor vector of $\left(\nu_{e}, \nu_{\mu}, \nu_{\tau}\right)_{\text {source }}$ changing 
to a flavor vector $\left(\nu_{e}, \nu_{\mu}, \nu_{\tau}\right)_{\text {Earth }}$ is given as

$$
\left(\begin{array}{c}
\nu_{e} \\
\nu_{\mu} \\
\nu_{\tau}
\end{array}\right)_{\text {Earth }}=\left(\begin{array}{lll}
0.534143 & 0.265544 & 0.200313 \\
0.265544 & 0.366436 & 0.368020 \\
0.200313 & 0.368020 & 0.431667
\end{array}\right)\left(\begin{array}{c}
\nu_{e} \\
\nu_{\mu} \\
\nu_{\tau}
\end{array}\right)_{\text {source }},
$$

for distances longer than the solar system.

\section{Dynamics of the Fireball}

Although we have already introduced general concepts of the fireball model and its dynamics, and also calculated the range of values of chemical potentials and temperatures for which the resonance condition is satisfied, here we are going to quantify or/and estimate the observable quantities in the evolution of the fireball requiring the charged-neutrality condition in addition to resonance condition. In this manner, we are going to constrain the dynamics of the fireball by means of neutrino oscillations.

\subsection{Initial stage}

The initial state of the fireball is magnetized, hot and dense of baryons and pairs $e^{ \pm}$in perfect thermodynamic equilibrium with a comoving temperature

$$
T^{\prime}=\left(\frac{L_{52}}{4 \pi r_{0,6}^{2} a}\right)^{1 / 4} \simeq 3.8 \mathrm{MeV}
$$


where $a=\pi^{2} k^{4} / 15=7.6 \times 10^{-15} \mathrm{erg} \mathrm{cm}^{-3} \mathrm{~K}^{-4}$ is the radiation density constant, $\mathrm{L}$ is the total isotropic-equivalent energy outflow in the jet and $r_{0}$ is the initial radius. Magnetic fields could be estimated by means of the equipartition of the total energy density (U)

$$
B \simeq \sqrt{8 \pi \epsilon_{B} U}
$$

which becomes important as the energy density is dominated by baryons (Beloborodov 2003a)

$$
U \simeq \frac{3}{2} \frac{T \rho}{m_{p}},
$$

and the electrons are degenerated with the charged-neutrality condition, $n_{e}(\mu, T, B)-$ $\bar{n}_{e}(\mu, T, B)=n_{p}$ where $n_{e}(\mu, T, B)$ and $\bar{n}_{e}(\mu, T, B)$ are the number densities of electrons and positrons generated in the plasma a temperature $\mathrm{T}$ and magnetic field $\mathrm{B}$, and $\mathrm{n}_{p}$ is the number density of protons. Taking into account that the number density of neutrons is comparable to that of protons and from the number density of electrons and positrons at the moderate magnetic field and relativistic temperatures found in section II (eq. B4), the changed-neutrality condition is given by

$$
\frac{m_{e}^{3}}{\pi^{2}} \Omega_{B} \sum_{l=0}^{\infty}(-1)^{l} \sinh \alpha_{l}\left\{K_{1}\left(\sigma_{l}\right)+2 \sum_{n=1}^{\infty} \lambda_{n} K_{1}\left(\sigma_{l} \lambda_{n}\right)\right\}=Y_{e} \frac{\rho}{m_{p}},
$$

here $\sigma_{l}$ and $\alpha_{l}$ correspond to the values found for which resonance condition is satisfied, $\rho$ is the baryon density and $Y_{e}=n_{p} /\left(n_{n}+n_{p}\right)$ is the proton-to-nucleon ratio. The baryon density is defined by means of the total mass outflow rate in baryon loaded jet $\dot{M}=4 \pi r_{0}^{2} \rho$ and the dimensionless entropy or baryon load parameter $\eta=L / \dot{M}$. The entropy per baryon is conserved in the flow and related to $\eta$ and $\mathrm{T}$ as $s / k_{B}=4 \eta m_{p} / 3 T$. Initially, due to high temperatures $T \gg m_{e}$ and charged current reactions

$$
e^{-}+p \rightarrow n+\nu, \quad e^{+}+n \rightarrow p+\bar{\nu},
$$

protons convert into neutrons and neutrons into protons, achieving a balance

$$
Y_{e}=\frac{1}{2}+\frac{7 \pi^{4}}{1350 \zeta(5)}\left(\frac{Q / 2-\mu}{T}\right)
$$


between the rates of $e^{-}$and $e^{+}$capture by $n_{p}$ and $n_{n}$ with Q defined through neutron $\left(m_{n}\right)$ and proton $\left(m_{p}\right)$ mass, $Q=m_{n}-m_{p}$ (Beloborodov 2003a). Hence, the changed-neutrality condition can be written as

$$
\sum_{l=0}^{\infty}(-1)^{l} \sinh \alpha_{l}\left\{K_{1}\left(\sigma_{l}\right)+2 \sum_{n=1}^{\infty} \lambda_{n} K_{1}\left(\sigma_{l} \lambda_{n}\right)\right\}=\frac{\rho \Omega_{B}^{-1}}{m_{p} m_{e}^{3}}\left[\frac{\pi^{2}}{2}+\frac{7 \pi^{6}}{1350 \zeta(5)}\left(\frac{Q / 2-\mu}{T}\right)\right] .
$$

It is very important to highlight that the proton-to-nucleon ratio is a function of temperature and chemical potential of the initial stage of the fireball, when the initial optical depth is extremely high and the phase of acceleration has not yet begun.

Taking into account the range of values of chemical potentials and temperatures found from resonance conditions (figs. 8 and 9), considering magnetic fields at the moderate field limit, $50 B_{c}$ (left-hand figures) and $0.1 B_{c}$ (right-hand figures) and requiring the charged-neutrality condition (eqs. 62 and 65), we plot contour lines of baryon density $(\rho)$ (above) and the proton-to-nucleon ratio $\left(\mathrm{Y}_{e}\right)$ (below) as a function of temperature as shown in fig. 11. From the figures above, one can see that the highest line of baryon density increases gradually achieving a maximum density of $7.2 \times 10^{7} \mathrm{~g} \mathrm{~cm}^{-3}$ (left-hand figure) and $5.6 \times 10^{6} \mathrm{~g} \mathrm{~cm}^{-3}$ (right-hand figure) for $2 \mathrm{MeV}$ $\leq T \leq 3 \mathrm{MeV}$ and $\mu=160 \mathrm{keV}$. This value of chemical potential is the largest one obtained from the resonance condition, hence resonant oscillations are suppressed for densities larger than these. From the figures below one can see that proton-to-nucleon ratio is a decreasing function of temperature achieving a minimum value of $\sim 0.52$ and 0.53 for baryon density $\simeq 10^{8} \mathrm{~g} \mathrm{~cm}^{-3}$ (left-hand figure) and $\simeq 10^{6} \mathrm{~g} \mathrm{~cm}^{-3}$ (right-hand figure) and maximum value of $\sim 0.87$ and 0.88 for baryon density $10^{6} \mathrm{~g} \mathrm{~cm}^{-3}$ (left-hand figure) and $10^{4.5} \mathrm{~g} \mathrm{~cm}^{-3}$, therefore resonant oscillations are only allowed when the number density of protons is at least slightly larger that that of neutrons $n_{p} \geq n_{n}$. This result could make evident the de-neutralization process in the fireball, where $Y_{e}$ is less than 0.5 at early times (there are not resonant oscillations) and after it becomes larger than 0.5 $\left(Y_{e}>0.5\right)$ at latter times (resonant oscillations are allowed). Other important characteristic from the figures below is that the number density of neutrons to protons ratio $n_{n} / n_{p}=\left(1-Y_{e}\right) / Y_{e}$ is lower for that fireball with more magnetization. For instance, taking the value of density $\rho=10^{6} \mathrm{~g} \mathrm{~cm}^{-3}$ 
in both plots, we can see that $Y_{e}\left(\mathrm{n}_{n} / \mathrm{n}_{p}\right)=0.865(0.156)$ for $\mathrm{B}=50 \mathrm{~B}_{c}$ and $Y_{e}\left(\mathrm{n}_{n} / \mathrm{n}_{p}\right)=0.751(0.332)$ for $\mathrm{B}=0.1 B_{c}$.

\subsection{Phase of acceleration}

When fireball starts expanding, the optically thick hot plasma expands with an increasing bulk Lorentz factor $\Gamma \propto r / r_{0}$ with radius $r$ following the adiabatic law and the comoving temperature drops as $T^{\prime}(r) \propto r_{0} / r$. Protons and neutrons keep coupled with each other until the value of Lorentz factor $\Gamma \simeq \eta$ is larger or less than a critical value or the dynamical time $t_{n p}^{\prime} \simeq(r / \Gamma)$ is shorter than the elastic scattering time $t_{n p}^{\prime} \simeq\left(n_{p}^{\prime} \sigma_{n p}\right)^{-1}$ where $\sigma_{n p} \approx 3 \times 10^{-26}$ $\mathrm{cm}^{2}$ and $n_{p}^{\prime}$ is the number density of protons. The critical value is defined by

$$
\eta_{\nu}=\left(\frac{L \sigma_{n p} Y_{e}}{4 \pi m_{p} r_{0}}\right)^{1 / 4} \simeq 4.6 \times 10^{2} L_{52}^{1 / 4} r_{o 7}^{-1 / 4} Y_{e}^{1 / 4}
$$

and depending on both $\eta$ and $\eta_{\nu}$ different processes of decoupling in a p-n outflow take place leading to varied energy ranges of neutrinos. For $\eta \geq \eta_{\nu}$, neutrinos in the energy range 5-10 $\mathrm{GeV}$ are expected (Bahcall \& meszaros 2000) whereas $\eta \leq \eta_{\nu}$ neutrino energy lies in the range 2-25 GeV or higher depending on the value of $\eta$ (Meszaros \& Rees 2000). Supposing that the decoupling takes place before coasting, then a slowly hadron shell $\left(\Gamma_{s}\right)$ is overtaken by another shell that in principle moves faster $\left(\Gamma_{f}=\eta\right)$ in the flow, producing inelastic collisions at $\mathrm{r} \sim 2 \Gamma_{s}^{2} r_{0}$. Following Meszaros \& Rees (2000), the typical collision Lorentz factor can be written as $\Gamma_{r e l} \simeq 1 / 2\left(\Gamma / \Gamma_{s}+\Gamma_{s} / \Gamma\right)$ with $\Gamma=\sqrt{\Gamma_{s} \Gamma_{f}} \simeq \Gamma_{f}$, and the total energy is $E_{\text {Tot }}=\left(2 m_{p}^{2}+2 m_{p} E_{r e l}\right)^{1 / 2}$, whereas the CM threshold energy is $2 m_{p}+m_{\pi} \sim 2 \mathrm{GeV}$. Hence, at depths $\tau_{p n} \sim n_{p}^{\prime} \sigma_{p n}(r / \Gamma)>1$ each neutron heated by an individual shock could collide $k_{\pi} \sim 2$ times before its $\mathrm{CM}$ energy becomes less than threshold. From the total number of neutrons involved in the shock $N_{n}=\left(1-Y_{e}\right) \epsilon E /\left(\eta m_{p}\right)$ and assuming a shock dissipation efficiency 
$\epsilon=0.2$, the number of expected events per year in DeepCore detector is

$$
R_{\nu \bar{\nu}} \sim 0.33 E_{53}\left(1-Y_{e}\right) N_{t 37.7} R_{b 3} h_{65}^{2}\left(\frac{2-\sqrt{2}}{1+z-\sqrt{1+z}}\right)^{2} \text { year }^{-1},
$$

where we have taken into account the product of density of ice and the effective volume at 100 $\mathrm{GeV}$ as $\rho_{\text {ice }} V_{\text {eff }} \simeq 50$ megaton $\left(V_{\text {eff }} \simeq 5.56 \times 10^{-2} \mathrm{~km}^{3}\right.$ and target protons of $\left.\mathrm{N}_{t} \sim 10^{37.7} N_{t 37.7}\right)$ (Abbasi \& et al. 2012a), a burst rate out to a Hubble radius of $10^{3} \mathrm{R}_{b}$ and an Einstein-de-Sitter universe with Hubble constant $\mathrm{H}=65 \mathrm{~h}_{65} \mathrm{~km} / \mathrm{s} / \mathrm{Mpc}$. Recently, Murase et al. (2013) showed that for a low-luminosity GRBs at $\mathrm{D}=10 \mathrm{Mpc}$ with $\Gamma=10$ and neutron luminosity $\mathrm{L}_{n}=2 \times 10^{46}$ erg/sec, quasi-thermal neutrinos around $10 \mathrm{GeV}$ are expected in DeepCore.

We plot the critical Lorentz factors $\left(\eta_{\nu}\right)$ (figure above) and number of neutrinos $\left(R_{\nu \nu}\right)$ (figure below) as a function of temperature when the resonance and charged-neutrality conditions are satisfied as shown in fig. 12. From figures above, one can see that the critical Lorentz factor is a decreasing function of temperature and decreasing function of baryon density. Besides, $\eta_{\nu}$ lies in the range $\simeq 436$ to 662 for temperatures and baryon densities in the range 50 to $10^{3} \mathrm{keV}$ and 10 to $10^{3}\left(1\right.$ to $\left.10^{2}\right) \mathrm{g} / \mathrm{cm}^{3}$ for $\mathrm{B}=10^{-4.5} B_{c}\left(\mathrm{~B}=10^{-5.5} B_{c}\right)$. From these plots can be seen that the effect of magnetic field is to increase $\eta_{\nu}$. In figures below, we can observe that the number of expected neutrinos is an increasing function of temperature and baryon density. Comparing both plots we see that as $\rho=10^{2} \mathrm{~g} / \mathrm{cm}^{3}$ and $\mathrm{T}=0.7 \mathrm{MeV}$ the expected neutrinos are $\simeq 2.23$ for $\mathrm{B}=10^{-4.3} B_{c}$ and $\simeq 4$ for $\mathrm{B}=10^{-5.3} B_{c}$, which tells us that the effect of magnetic field is to decrease the number of expected neutrinos. From that we can see the importance of knowing the strength of magnetic field and as it could alter the dynamics.

It is important to notice that independently of the model and considerations assumed (Bahcall \& meszaros 2000; Meszaros \& Rees 2000; Murase et al. 2013) multi-GeV neutrinos are expected on Earth, hence we will estimate their flavor ratio. Considering the flux ratio for $\pi^{ \pm}$and $\mu^{ \pm}$decay as $\dot{N}_{\nu_{\mu}} \simeq \dot{N}_{\bar{\nu}_{\mu}} \simeq 2 \dot{N}_{\nu_{e}} \simeq 2 \dot{N}_{\bar{\nu}_{e}}($ Razzaque \& Meszaros 2006a) and from oscillation probabilities at $10^{11} \mathrm{~cm}, 10^{12} \mathrm{~cm}$ and $10^{13} \mathrm{~cm}$ given in the section III, we compute the flavor ratio for neutrino energies of 5,10, 20 and $50 \mathrm{GeV}$ as shown in table 3. From this table one can see an interesting 
result, that although tau neutrino at $\mathrm{GeV}$ energies is not created by p-n decoupling, it appears due to the resonant oscillations of muon neutrinos in the fireball. In addition, from table 3 and eq. 58 we estimate the flavor ratio expected on Earth for the same range of neutrino energy as shown in table 4. As shown in this table, $\mathrm{GeV}$ sub-photospheric neutrinos with deviations of this standard flavor ratios are expected.

\section{Results and Conclusions}

We have explicitly calculated the neutrino self-energy and neutrino effective potential up to an order $m_{W}^{-4}$ as a function of temperature, chemical potential, magnetic field and neutrino energy. We have shown that for neutrinos in the $\mathrm{GeV}$ energy range as well as small chemical potentials which is the case of solar and atmospheric neutrino parameter (small particle-antiparticle asymmetry) the contribution of $\mathrm{m}_{W}^{-4}$ terms to the neutrino effective potential is relevant. In the magnetic field framework, we have derived it at the strong, moderate (above and below $\mathrm{B}_{c}$ ) and weak-field limit, taking into account a background composed of pairs $e^{ \pm}$, photons, protons, neutrons and neutrinos. Also we have derived the resonance and charged-neutrality conditions. By considering the neutrino effective potential at the moderate field limit, using the typical values of a magnetized GRB fireball and requiring the resonance and charged-neutrality conditions, we have studied the thermal and quasi-thermal neutrino oscillations assuming a neutron abundance which is comparable to that of protons. In the fireball scenario, thermal neutrino oscillations have been studied at the initial stage $\left(r_{0} \simeq 10^{6.5}-10^{7.5} \mathrm{~cm}, B \simeq 0.1-50 B_{c}\right.$ and $T \simeq 1-10) \mathrm{MeV}$ whereas quasi-thermal $\mathrm{GeV}$ neutrinos in the phase of acceleration $\left(\mathrm{r} \simeq 10^{11}-10^{13} \mathrm{~cm}, B \simeq 10^{-4.3}-10^{-5.3} B_{c}\right.$ and $\left.T \simeq 50-700 \mathrm{keV}\right)$. This complete analysis has been carried out using the two- (solar, atmospheric and accelerator neutrino parameters) and three-neutrino mixing.

The results for the initial stage are: 


\begin{tabular}{|c|c|c|c|}
\hline $\begin{array}{c}E_{\nu} \\
(\mathrm{GeV})\end{array}$ & $\begin{array}{c}\phi_{\nu_{e}}: \phi_{\nu_{\mu}}: \phi_{\nu_{\tau}} \\
\left(\mathrm{r}=10^{11} \mathrm{~cm}\right)\end{array}$ & $\begin{array}{c}\phi_{\nu_{e}}: \phi_{\nu_{\mu}}: \phi_{\nu_{\tau}} \\
\left(\mathrm{r}=10^{12} \mathrm{~cm}\right)\end{array}$ & $\begin{array}{c}\phi_{\nu_{e}}: \phi_{\nu_{\mu}}: \phi_{\nu_{\tau}} \\
\left(\mathrm{r}=10^{13} \mathrm{~cm}\right)\end{array}$ \\
\hline 5 & $1.000: 1.959: 0.041$ & $1.000: 0.021: 1.979$ & $1.000: 0.848: 1.152$ \\
\hline 10 & $1.000: 0.164: 1.837$ & $1.000: 0.951: 1.049$ & $1.000: 0.000: 2.000$ \\
\hline 20 & $1.000: 1.101: 0.899$ & $1.000: 0.282: 1.718$ & $1.000: 1.917: 0.083$ \\
\hline 50 & $1.000: 0.675: 1.325$ & $1.000: 0.002: 1.998$ & $1.000: 1.839: 0.162$ \\
\hline
\end{tabular}

Table 3: The flavor ratio on the surface of the fireball for four neutrino energies $\left(E_{\nu}=5 \mathrm{GeV}, 10 \mathrm{GeV}\right.$, $20 \mathrm{GeV}$ and $50 \mathrm{GeV}$ ), leaving the star to three distances $r=10^{11} \mathrm{~cm}, 10^{12} \mathrm{~cm}$ and $10^{13} \mathrm{~cm}$.

\begin{tabular}{|c|c|c|c|}
\hline $\begin{array}{c}E_{\nu} \\
(\mathrm{TeV})\end{array}$ & $\begin{array}{c}\phi_{\nu_{e}}^{0}: \phi_{\nu_{\mu}}^{0}: \phi_{\nu_{\tau}}^{0} \\
\left(\mathrm{r}=10^{11} \mathrm{~cm}\right)\end{array}$ & $\begin{array}{c}\phi_{\nu_{e}}^{0}: \phi_{\nu_{\mu}}^{0}: \phi_{\nu_{\tau}}^{0} \\
\left(\mathrm{r}=10^{12} \mathrm{~cm}\right)\end{array}$ & $\begin{array}{c}\phi_{\nu_{e}}^{0}: \phi_{\nu_{\mu}}^{0}: \phi_{\nu_{\tau}}^{0} \\
\left(\mathrm{r}=10^{13} \mathrm{~cm}\right)\end{array}$ \\
\hline 5 & $1.063: 0.998: 0.939$ & $0.936: 1.002: 1.062$ & $0.990: 1.000: 1.010$ \\
\hline 10 & $0.945: 1.001: 1.053$ & $0.997: 1.000: 1.003$ & $0.935: 1.002: 1.064$ \\
\hline 20 & $1.007: 0.999: 0.994$ & $0.953: 1.001: 1.046$ & $1.060: 0.999: 0.941$ \\
\hline 50 & $0.979: 1.001: 1.021$ & $0.935: 1.002: 1.064$ & $1.055: 0.999: 0.947$ \\
\hline
\end{tabular}

Table 4: The flavor ratio expected on Earth for four neutrino energies $\left(\mathrm{E}_{\nu}=5 \mathrm{GeV}, 10 \mathrm{GeV}, 20 \mathrm{GeV}\right.$ and $50 \mathrm{GeV}$ ), leaving the star to three distances $r=10^{11} \mathrm{~cm}, 10^{12} \mathrm{~cm}$ and $10^{13} \mathrm{~cm}$. 
1. Resonant oscillations are suppressed for baryon densities greater than $\sim 10^{8} \mathrm{~g} \mathrm{~cm}^{-3}$ for 50 $B_{c}$ and $\sim 10^{6} \mathrm{~g} \mathrm{~cm}^{-3}$ for $0.1 B_{c}$.

2. Neutrinos can hardly oscillate resonantly for a fireball with number density of neutrons greater than protons $n_{n} \geq n_{p}$ or for proton-to-baryon ratio larger than $0.52\left(Y_{e} \leq 0.52\right)$.

3. The number density of neutrons to protons ratio $\left(n_{n} / n_{p}\right)$ is lower for that fireball with more magnetization. It is due to positron capture on neutrons is greatly accelerated by the large magnetic phase space factor (Thompson \& Gill 2013). Also baryon densities are larger for a fireball with more magnetization.

From the phase of acceleration, we showed that:

1. The critical Lorentz factor for neutrino production is limited by the load density, temperature and the magnetic field. The effect of magnetic field in the emission region is to decrease the expected number of neutrinos.

2. GeV neutrinos created in the sub-photospheric region can oscillate resonantly. Due to this, we estimate the neutrino flavor ratio and deviations of standard flavor ratios are expected.

Neutrinos of energies of 1 to $30 \mathrm{MeV}$ are very similar to those produced by type II supernova i.e, SN1987A, however are of cosmological distance. These cosmological events make the thermal neutrino flux very low on Earth compared to the ones we had seen from the supernova SN1987A. 
Although with the current neutrino telescopes, low energy neutrinos would be pretty difficult to detect, such survey would help us to understand the dynamics of the jet as it changes with the content of baryons.

We thank the anonymous referee for a critical reading of the paper and valuable suggestions that helped improve the quality and clarity of this work. We also thank Bing Zhang, Kohta Murase, Ignacio Taboada, William Lee, Dany Page and Enrique Moreno for useful discussions. This work was supported by Luc Binette scholarship and the projects IG100414 and Conacyt 101958. 


\section{REFERENCES}

Abbasi R., \& et al. 2012a, ApJ, 35, 615

Abbasi R., \& et al. 2012b, Nature, 484, 315

Abe, K., Hayato, Y. et al., 2011, Phys. Rev. D. , 107, 241801

Aharmin, B., Ahmed, S. N. et al., 2011, arXiv:1109.0763v1

Akhmedov E. K. , Johansson R., Lindner M., Ohlsson T. and Schwetz T. , 2004, JHEP, 0404, 078

Athanassopoulos, C. et al., 1996, arXiv:9605003v1

Athanassopoulos, C. et al. , 1998, Phys. Rev. Lett., 81, 9

Bahcall, J. N. and Mészáros, P. 2000, Phys. Rev. Lett., 85, 1362

Beloborodov, A. M. 2003, ApJ585, L19

Beloborodov, A. M. 2003, ApJ588, 931

Blandford \& Znajek 1977, MNRAS179, 433

Blandford R. D. , 2002, astro-ph/0202265

Boggs S. E. and Coburn W., (2003), MNRAS, submitted, astro-ph/0310515

Bravo. Garcia A. and Sahu S., 2007, Mod. Phys. Lett. A, 22213

Church E.D. et al. , 2002, Phys. Rev. D., 66, 013001

Coburn W. and Boggs S. E., 2003, Nature, 423, 415

B. Dasgupta, A. Dighe, A. Mirizzi and G. G. Raffelt , 2008, Phys. Rev. D. 78, 033014

Derishev, E. V., Kocharovsky, V. V. and Kocharovsky, VI. V. 1999a, ApJ, 521, 640 
Derishev, E. V., Kocharovsky, V. V. and Kocharovsky, VI. V. 1999b, A\&A, 345, L51

Dermer, C. D. and Atoyan, A. 2006, ApJ2006 643 L13

Dermer, C. D., Razzaque, S., Finke, J. D., \& Atoyan, A. 2009, New Journal of Physics, 11, 065016

D’Olivo J.C., Nieves J. F. and Torres M., 1992, Phys. Rev. D. 46, 1172

D’Olivo J.C. \& Nieves J. F. , 1994, Nucl. Phys. B 35, 466

D’Olivo J.C. \& Nieves J. F. , 1996, International Journal of Modern Physics A 11, 141

D’Olivo J.C. \& Nieves J. F. , 1996, Phys. Letters B 383, 87

D’Olivo J.C. \& Nieves J. F. , 1997, Phys. Rev. D. 56, 5898

D’Olivo J.C., Nieves J. F. and Sahu S., 2003, Phys. Rev. D. 67, 025018

Elizalde E., Ferrer E.J. \& Incera V., 2004, Phys. Rev. D., 70, 0430012

Engel, R. 2008, in International Cosmic Ray Conference, Vol. 4, International Cosmic Ray Conference, $385-388$

Enquist K., Kainulainen K. \& Maalampi J. 1991, Nucl. Phys. B 349, 754

Erdas A. and Feldman G., 1990, Nucl. Phys. B 343, 597

Erdas A., Kim C. W. and Lee T. H., 1998, Phys. Rev. D, 58, 085016

Erdas A. and Isola C., 2000, Phys. Letter B. 494, 262

Erdas A., 2009, Phys. Rev. D. 80, 3004

Fan, Y. Z. and Wei, D. M. 2004, ApJ615, L69 
Fan, Y. Z., Zhang, B. and Wei, D. M. 2005, ApJ, 628, L25

Fraija N., Gonzalez M. M., Lee W. H. 2012, ApJ751, 33

Fraija N., Gonzalez M. M., Sacahui R. and Lee W. H. 2012, PO in Science 27, arXiv:1212.4418

Fraija N. 2014, MNRAS437, 2187

Gonzalez-Garcia, M. C., 2011, Physics of Particles and Nuclei, 42, 4

Gonzalez-Garcia, M. C., 2002, Review of Modern Physics, 75, 2

Gonzalez-Garcia, M. C. \& Maltoni, M., 2008, Phys. Rep., 460, 1

Goodman J ,Dar A. and Nussinov S., 1987, ApJ314, L7

Janka H.T. et al., 2012, arXiv:1211.1378

Koers, H. B. J. and Wijers, R. A. M. J. 2005, MNRAS364 934

Koers, H. B. J.; Giannios, D. A\&A2007 471395

Learned J. G., Pakvasa S., 1995, Astroparticle Physics, 3, 267

Lee H. K., Wijers R.A.M.J. and Brown G. E., 2000, Phys. Rep., 325, 83

Lyutikov M. and Blandford R. , 2002, astro-ph/0210671

Mészáros, P. \& Rees, M. J., 1994, MNRAS, 269, L41

Mészáros, P. \& Rees, M. J., 1997a, ApJ, 476, 232

Mészáros, P. \& Rees, M. J., 1997b, ApJ, 482, L29

Metzger et al., 2010, arXiv:2012.0001

Metzger, Brian D.; Thompson, Todd A.; Quataert, Eliot 20086761130 
Mészáros, P. 2006, Report of Progress in Physics, 69, 2259

Mészáros, P. \& Rees, M. J., 1997b, ApJ, 482, L29

Mészáros, P. and Rees, M. J., 2000, ApJ541, L5

Mészáros, P. 2012, Astrop. Phys. arXiv:1204.1897

Michel, F. C. 1969, Nature, 223, 277

Murase K., Kashiyama K. and Mészáros P., 2013, Phys. Rev. Lett. 111131102

Nieves J. F., 1990, Phys. Rev. D 42, 4123 [Erratum-ibid. D 49, 3067 (1994)].

Nötzold D. \& Raffelt G. 1988, Nucl. Phys. B 307, 924

Paczyński B. \& Rhoads J., 1993, ApJ, 418, L5

Putten M. H. P. M., 2001, Phys. Rep., 345, 1

Razzaque, Soebur; Mszros, Peter JCAP 2006a 06006

Razzaque, S. and Meszaros, P. 2006, ApJ2006b 650998

Rees, M. J. \& Mészáros, P., 1994, ApJ, 430, L93

Rossi, Elena M.; Beloborodov, Andrei M.; Rees, Martin J. MNRAS2006, 369, 1797

Ruffert M. and Janka H.T., 1999, A\&A344, 573

Rutledge R. E. \& Fox D. B., 2004, , 350, 1288

Sacahui R., Fraija N., Gonzalez M. M. and Lee W. H. 2012, ApJ, 755, 127

Sahu S. and D’Olivo J. C., 2005, Phys. Rev. D. 71, 047303

Sahu S.,Fraija N. and Keum Y. Y., 2009, Phys. Rev. D, 80, 033009 
Sahu S.,Fraija N. and Keum Y. Y., 2009, JCAP 11, 024

Sahu S., Zhang B. and Fraija N., 2012, Phys. Rev. D. 85, 043012

Schwinger J,, 1951, Phys. Rev. Lett. 82, 5, 664

Spruit H. C. and Drenkhahn G., 2003, astro-ph/0302468

Thompson C. \& Gill R. 2013, astro-ph/1310.2480

Tututi, E. S. and Torres, M. and D’Olivo, J. C., 2002, Phys. Rev. D. 66, 3001

Usov V. V., 1992, Nature, 357, 472

Usov V. V., in Gamma-ray bursts: The First Three Minutes, ed. J. Poutanen and R. Svensson (ASP Conf. Ser. V109), 153 (1999)

Vlahakis N. and A. Königl, 2003, ApJ596 1080

N. Vlahakis and A. Königl, 2003, ApJ596, 1104

Volkas R.R. and Wong Y.Y.Y., 2000, Astropart. Phys. 13, 21

Weldon H. A, 1982., Phys. Rev. D., 26, 1394.

Wendell, R., Ishihara, C. et al., 2010, Phys. Rev. D. v , 81, 092004

Wheeler J. C., Yi I., Hoflitch P. and Wang L. , 2000, ApJ537, 810

Wolfenstein, 1978, Phys. Rev. D., 17, 2369

Xue, Rong-Rong; Fan, Yi-Zhong; Wei, Da-Ming MNRAS2008 389321

Zhang B. \& Meszaros P., 2002, ApJ, 581, 1236

Zhang, B., Kobayashi, S. \& Mészáros, P. 2003 ApJ, 595, 950 
Zhang, B. \& Mészáros, P. 2004, Int. J. Mod. Phys., A19, 2385

Zhang, B.\& Kobayashi, S. 2005 ApJ, 628, 315

Zhang, B.\& Yan, H. 2011, ApJ726, 90

Zhang, B.\& Kumar, P. 2013, Phys. Rev. Lett. 110, 121101 


\section{A. Strong Magnetic field: $\Omega_{B} \gg 1$}

In the strong magnetic field approximation, the energy of charged particles is modified confining the particles to the lowest Landau level $(n=0)$. Thus, the number density of electrons given by Eq. (23) will become

$$
n_{e}^{0}=\frac{e B}{2 \pi^{2}} \int_{0}^{\infty} d p_{3} f_{e, 0},
$$

where

$$
f\left(E_{e, 0}\right)=\frac{1}{e^{\beta\left(E_{e, 0}-\mu\right)}+1},
$$

and the electron energy in the lowest Landau level is,

$$
E_{e, 0}^{2}=\left(p_{3}^{2}+m_{e}^{2}\right) .
$$

Assuming that the chemical potentials $(\mu)$ of the electrons and positrons are smaller than their energies $\left(\mu \leq \mathrm{E}_{e}\right)$, the fermion distribution function can be written as a sum given by

$$
f\left(E_{e, 0}\right)=\frac{1}{e^{\beta\left(E_{e, 0}-\mu\right)}+1} \approx \sum_{l=0}^{\infty}(-1)^{l} e^{-\beta\left(E_{e, 0}-\mu\right)(l+1)} .
$$

Therefore, the Lorentz scalars in this approximation are reduced to (Elizalde et al. 2004; Sahu et al. 2009a)

$$
\begin{aligned}
b_{W}=\quad & \sqrt{2} G_{F}\left[\left(1+\frac{3}{2} \frac{m_{e}^{2}}{m_{W}^{2}}+\frac{e B}{m_{W}^{2}}+\frac{E_{\nu_{e}} k_{3}}{m_{W}^{2}}+\frac{E_{\nu_{e}}^{2}}{m_{W}^{2}}\right)\left(N_{e}^{0}-\bar{N}_{e}^{0}\right)\right. \\
& \left.-\frac{e B}{2 \pi^{2} m_{W}^{2}} \int_{0}^{\infty} d p_{3}\left\{2 k_{3} E_{e, 0}+2 E_{\nu_{e}}\left(E_{e, 0}-\frac{m_{e}^{2}}{2 E_{e, 0}}\right)\right\}\left(f_{e, 0}+\bar{f}_{e, 0}\right)\right]
\end{aligned}
$$

and

$$
\begin{aligned}
c_{W}= & \sqrt{2} G_{F}\left[\left(1+\frac{1}{2} \frac{m_{e}^{2}}{m_{W}^{2}}+\frac{e B}{m_{W}^{2}}-\frac{E_{\nu_{e}} k_{3}}{m_{W}^{2}}-\frac{k_{3}^{2}}{m_{W}^{2}}\right)\left(N_{e}^{0}-\bar{N}_{e}^{0}\right)\right. \\
& \left.\left.-\frac{e B}{2 \pi^{2} m_{W}^{2}} \int_{0}^{\infty} d p_{3}\left\{2 E_{\nu_{e}}\left(E_{e, 0}-\frac{m_{e}^{2}}{2 E_{e, 0}}\right)\right\}+2 k_{3}\left(E_{e, 0}-\frac{3 m_{e}^{2}}{2 E_{e, 0}}\right)\right\}\left(f_{e, 0}+\bar{f}_{e, 0}\right)\right] \text { (A6) }
\end{aligned}
$$

For Z-exchange diagram we do not have magnetic contribution and for the tadpole diagram only electron loop will be affected by the magnetic field (Sahu et al. 2009b). The electron number 
density and other useful quantities at the strong field limit are

$$
\begin{gathered}
N_{e}^{0}=\frac{m^{3}}{2 \pi^{2}} \frac{B}{B_{c}} \sum_{l=0}^{\infty}(-1)^{l} e_{l}^{\alpha} K_{1}\left(\sigma_{l}\right), \\
N_{e}^{0}-\bar{N}_{e}^{0}=\frac{B}{B_{c}} \frac{m_{e}^{3}}{\pi^{2}} \sum_{l=0}^{\infty}(-1)^{l} \sinh \alpha_{l} K_{1}\left(\sigma_{l}\right), \\
\frac{e B}{2 \pi^{2}} \int_{0}^{\infty} d p_{3} E_{0}\left(f_{e, 0}+\bar{f}_{e, 0}\right)=\frac{m_{e}^{4}}{\pi^{2}} \frac{B}{B_{c}} \sum_{l=0}^{\infty}(-1)^{l} \cosh \alpha_{l}\left(K_{0}\left(\sigma_{l}\right)+\frac{K_{1}\left(\sigma_{l}\right)}{\sigma_{l}}\right), \\
\frac{e B}{2 \pi^{2}} \int_{0}^{\infty} d p_{3} \frac{1}{E_{0}}\left(f_{e, 0}+\bar{f}_{e, 0}\right)=\frac{m_{e}^{4}}{\pi^{2}} \frac{B}{B_{c}} \sum_{l=0}^{\infty}(-1)^{l} \cosh \alpha_{l} K_{0}\left(\sigma_{l}\right),
\end{gathered}
$$

where we have defined

$$
\alpha_{l}=\beta \mu(l+1) \quad \text { and } \quad \sigma_{\mathrm{l}}=\beta \mathrm{m}_{\mathrm{e}}(1+1)
$$

with $\mathrm{K}_{i}$ is the modified Bessel function of integral order i. Replacing Eqs. (A8) and (A9) in the Lorentz scalars (Eqs. A5 and A6), we obtain

$$
\begin{aligned}
b_{W}= & \frac{\sqrt{2} G_{F} m_{e}^{3}}{\pi^{2}} \frac{B}{B_{c}}\left[\left\{1+\frac{m_{e}^{2}}{m_{W}^{2}}\left(\frac{3}{2}+2 \frac{E_{\nu, e}^{2}}{m_{e}^{2}}+\frac{B}{B_{c}}\right)\right\} \sum_{l=0}^{\infty}(-1)^{l} \sinh \alpha_{l} K_{1}\left(\sigma_{l}\right)\right. \\
& \left.-\frac{m_{e}^{2}}{m_{W}^{2}} \frac{E_{\nu, e}}{m_{e}} \sum_{l=0}^{\infty}(-1)^{l} \cosh \alpha_{l}\left\{3 K_{0}\left(\sigma_{l}\right)+4 \frac{K_{1}\left(\sigma_{l}\right)}{\sigma_{l}}\right\}\right], \\
c_{W}= & \frac{\sqrt{2} G_{F} m_{e}^{3}}{\pi^{2}} \frac{B}{B_{c}}\left[\left\{1+\frac{m_{e}^{2}}{m_{W}^{2}}\left(\frac{1}{2}-2 \frac{E_{\nu, e}^{2}}{m_{e}^{2}}+\frac{B}{B_{c}}\right)\right\} \sum_{l=0}^{\infty}(-1)^{l} \sinh \alpha_{l} K_{1}\left(\sigma_{l}\right)\right. \\
& \left.-4 \frac{m_{e}^{2}}{m_{W}^{2}} \frac{E_{\nu, e}}{m_{e}} \sum_{l=0}^{\infty}(-1)^{l} \cosh \alpha_{l} \frac{K_{1}\left(\sigma_{l}\right)}{\sigma_{l}}\right] .
\end{aligned}
$$

Finally, from Eqs. (6), (A11) and (A12) and for neutrinos moving along the direction of magnetic field, the neutrino effective potential in the strong magnetic field regime is

$$
\begin{aligned}
V_{e f f}= & \frac{\sqrt{2} G_{F} m_{e}^{3}}{\pi^{2}} \frac{B}{B_{c}}\left[\sum _ { l = 0 } ^ { \infty } ( - 1 ) ^ { l } \operatorname { s i n h } \alpha _ { l } K _ { 1 } ( \sigma _ { l } ) \left\{1+\frac{m_{e}^{2}}{m_{W}^{2}}\left(\frac{3}{2}+2 \frac{E_{\nu}^{2}}{m_{e}^{2}}+\frac{B}{B_{c}}\right)\right.\right. \\
& \left.-\left(1+\frac{m_{e}^{2}}{m_{W}^{2}}\left(\frac{1}{2}-2 \frac{E_{\nu}^{2}}{m_{e}^{2}}+\frac{B}{B_{c}}\right)\right) \cos \phi\right\} \\
& \left.-4 \frac{m_{e}^{2}}{m_{W}^{2}} \frac{E_{\nu}}{m_{e}} \sum_{l=0}^{\infty}(-1)^{l} \cosh \alpha_{l}\left\{\frac{3}{4} K_{0}\left(\sigma_{l}\right)+\frac{K_{1}\left(\sigma_{l}\right)}{\sigma_{l}}-\frac{K_{1}\left(\sigma_{l}\right)}{\sigma_{l}} \cos \phi\right\}\right] .
\end{aligned}
$$

Doing $\Omega_{B}=B / B_{c}$ and the previous effective potential is written as shown in eq. (29). 


\section{B. Moderate Magnetic field: $\Omega_{B}>1$ and $\Omega_{B} \leq 1$}

The electron energy in the magnetic field is given by

$$
E_{e, n}^{2}=\left(p_{3}^{2}+m_{e}^{2}+2 n e B\right)=p_{3}^{2}+m_{e}^{2}\left(1+2 n \Omega_{B}\right)
$$

In this case, the number density of electrons (eq. 23) is written as

$$
n_{e}=\frac{\Omega_{B} m_{e}^{2}}{2 \pi^{2}}\left[\int_{0}^{\infty} d p_{3} f_{e, 0}+2 \sum_{n=1}^{\infty} \int_{0}^{\infty} d p_{3} f_{e, n}\right]
$$

and the electron distribution function by

$$
f\left(E_{e, n}\right)=\frac{1}{e^{\beta\left(E_{e, n}-\mu\right)}+1} \approx \sum_{l=0}^{\infty}(-1)^{l} e^{-\beta\left(E_{e, n}-\mu\right)(l+1)} .
$$

Calculating the below useful quantities in this regime,

$$
\begin{gathered}
n_{e}-\bar{n}_{e}=\frac{m^{3}}{\pi^{2}} \Omega_{B}\left[\sum_{l=0}^{\infty}(-1)^{l} \sinh \alpha_{l}\left\{K_{1}\left(\sigma_{l}\right)+2 \sum_{n=1}^{\infty} \lambda_{n} K_{1}\left(\sigma_{l} \lambda_{n}\right)\right\}\right] \\
n_{e}+\bar{n}_{e}=\frac{m^{3}}{\pi^{2}} \Omega_{B}\left[\sum_{l=0}^{\infty}(-1)^{l} \cosh \alpha_{l}\left\{K_{1}\left(\sigma_{l}\right)+2 \sum_{n=1}^{\infty} \lambda_{n} K_{1}\left(\sigma_{l} \lambda_{n}\right)\right\}\right] \\
\int_{n}^{\infty} d p_{3} E_{n}\left(f_{e, n}+\bar{f}_{e, 0}\right)=2 m_{e}^{2} \sum_{l=0}^{\infty}(-1)^{l} \cosh \alpha_{l}\left[\left(K_{0}\left(\sigma_{l}\right)+\frac{K_{1}\left(\sigma_{l}\right)}{\sigma_{l}}\right)+2 \sum_{n=1}^{\infty} \lambda_{n}^{2}\left(K_{0}\left(\sigma_{l} \lambda_{n}\right)+\frac{K_{1}\left(\sigma_{l} \lambda_{n}\right)}{\sigma_{l}}\right)\right] \\
\int_{0}^{\infty} d p_{3} \frac{1}{E_{n}}\left(f_{e, n}+\bar{f}_{e, n}\right)=2 \sum_{l=0}^{\infty}(-1)^{l} \cosh \alpha_{l}\left[K_{0}\left(\sigma_{l}\right)+\frac{1}{2} \sum_{n=1}^{\infty} K_{0}\left(\sigma_{l} \lambda_{n}\right)\right]
\end{gathered}
$$

where $\lambda$ is defined by

$$
\lambda^{2}=\left\{\begin{array}{lll}
2 n \Omega_{B} & \text { for } \Omega_{B}>1 & \text { moderately above } \\
1+2 n \Omega_{B} & \text { for } \Omega_{B} \leq 1 & \text { moderately below }
\end{array}\right.
$$

with the Lorentz scalars and again with $A_{e}=\sqrt{2} G_{F} \frac{m_{e}^{3} \Omega_{B}}{\pi^{2}}$, the neutrino effective potential in the moderate regimen can be written as shown in eq. (30). 


\section{Weak Magnetic field: $\Omega_{B} \ll 1$}

In this regimen, sums over the parameter $\lambda=\sqrt{1+2 n \Omega_{B}}$ can be approximated by an integral $\sum_{n} \rightarrow \int d n$. Therefore, it is useful to write the following relations

$$
\begin{aligned}
\sum_{n=0}^{\infty} \lambda_{n}^{2} K_{0}\left(\sigma_{l} \lambda_{n}\right) & =\Omega_{B}^{-1}\left[2 \frac{K_{0}\left(\sigma_{l}\right)}{\sigma_{l}}+\left(1+\frac{4}{\sigma_{l}^{2}}\right) \frac{K_{1}\left(\sigma_{l}\right)}{\sigma_{l}}\right] \\
\sum_{n=0}^{\infty} \lambda_{n} K_{1}\left(\sigma_{l} \lambda_{n}\right) & =\Omega_{B}^{-1}\left[\frac{K_{0}\left(\sigma_{l}\right)}{\sigma_{l}}+\frac{2}{\sigma_{l}^{2}} K_{1}\left(\sigma_{l}\right)\right]
\end{aligned}
$$

where we have applied the integrals

$$
\begin{aligned}
\int_{1}^{\infty} \frac{t^{2} d t}{\sqrt{t^{2}-1}} e^{-\sigma_{l} t} & =K_{0}\left(\sigma_{l}\right)+\frac{K_{1}\left(\sigma_{l}\right)}{\sigma_{l}} \\
\int_{1}^{\infty} \frac{t d t}{\sqrt{t^{2}-1}} e^{-\sigma_{l} t} & =K_{1}\left(\sigma_{l}\right) \\
\int_{1}^{\infty} \frac{d t}{\sqrt{t^{2}-1}} e^{-\sigma_{l} t} & =K_{0}\left(\sigma_{l}\right)
\end{aligned}
$$

for $R e \sigma_{l}>0$ and we have used the recurrence relation $K_{3}\left(\sigma_{l}\right)=\frac{4}{\sigma_{l}} K_{0}\left(\sigma_{l}\right)+\left(1+\frac{8}{\sigma_{l}^{2}}\right) K_{1}\left(\sigma_{l}\right)$ and $K_{2}\left(\sigma_{l}\right)=K_{0}\left(\sigma_{l}\right)+\frac{2}{\sigma_{l}} K_{1}\left(\sigma_{l}\right)$. Therefore, from eqs. (B4), $\left.\mathrm{B} 5\right)$ and 30 , the neutrino effective potential is given by eq. (31) and useful functions of electron number density can be written as

$$
\begin{gathered}
n_{e}-\bar{n}_{e}=\frac{m^{3}}{\pi^{2}} \Omega_{B}\left[2 \sum_{l=0}^{\infty}(-1)^{l} \sinh \alpha_{l}\left\{\left(\frac{K_{0}\left(\sigma_{l}\right)}{\sigma_{l}}+\frac{K_{1}\left(\sigma_{l}\right)}{\sigma_{l}^{2}}\right) \Omega_{B}^{-1}-\frac{1}{2} K_{1}\left(\sigma_{l}\right)\right\}\right] \\
n_{e}+\bar{n}_{e}=\frac{m^{3}}{\pi^{2}} \Omega_{B}\left[2 \sum_{l=0}^{\infty}(-1)^{l} \cosh \alpha_{l}\left\{\left(\frac{K_{0}\left(\sigma_{l}\right)}{\sigma_{l}}+\frac{K_{1}\left(\sigma_{l}\right)}{\sigma_{l}^{2}}\right) \Omega_{B}^{-1}-\frac{1}{2} K_{1}\left(\sigma_{l}\right)\right\}\right] .
\end{gathered}
$$



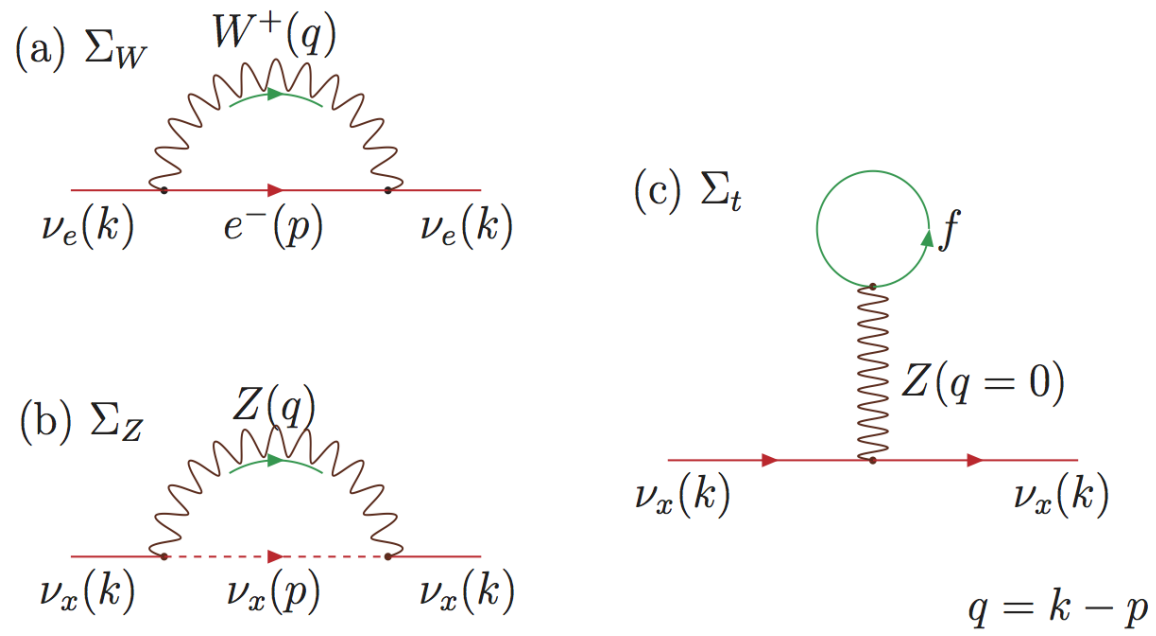

Fig. 1.- One-loop contribution to the neutrino self-energy in a magnetized medium. a) Wexchange diagram: The solid line represents the electron propagator and the wiggly line the $\mathrm{W}$ boson in a magnetized medium. b) Z-exchange diagram: The dashed line corresponds to the neutrino propagator in a thermal medium and the wiggly line to the Z-boson c) Tadpole diagram: The solid line represents the fermion propagator and the wiggly line the Z-boson in a magnetized medium. 

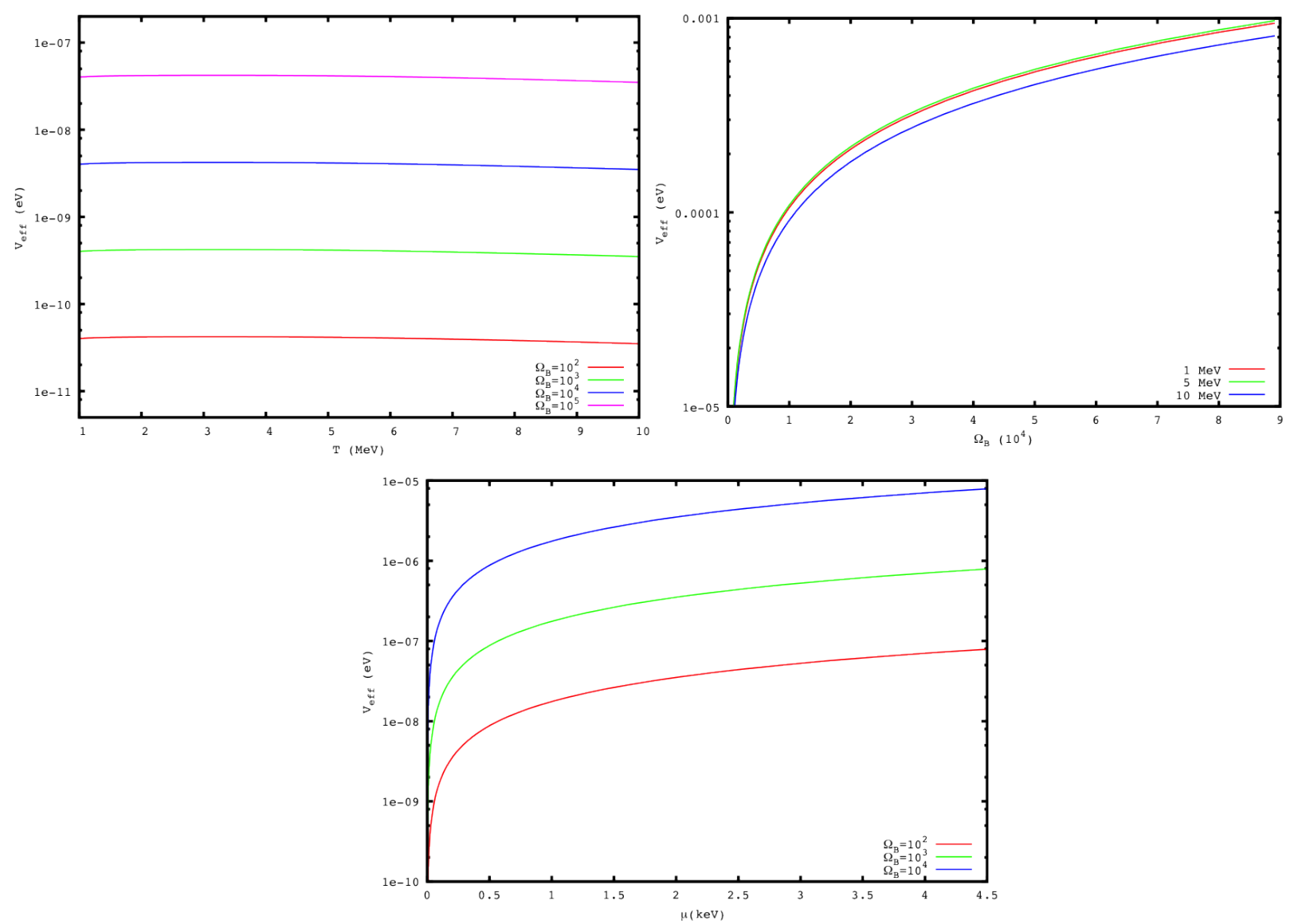

Fig. 2.- Neutrino effective potential in the strong magnetic field regime as a function of temperature (T) (left-hand figure above), magnetic field $\left(\Omega_{B}\right)$ (right-hand figure above), chemical potential $(\mu)$ (figure below) and neutrino energy $\left(\mathrm{E}_{\nu}\right)$. All plots are obtained for a neutrino energy of 10 $\mathrm{MeV}$. 

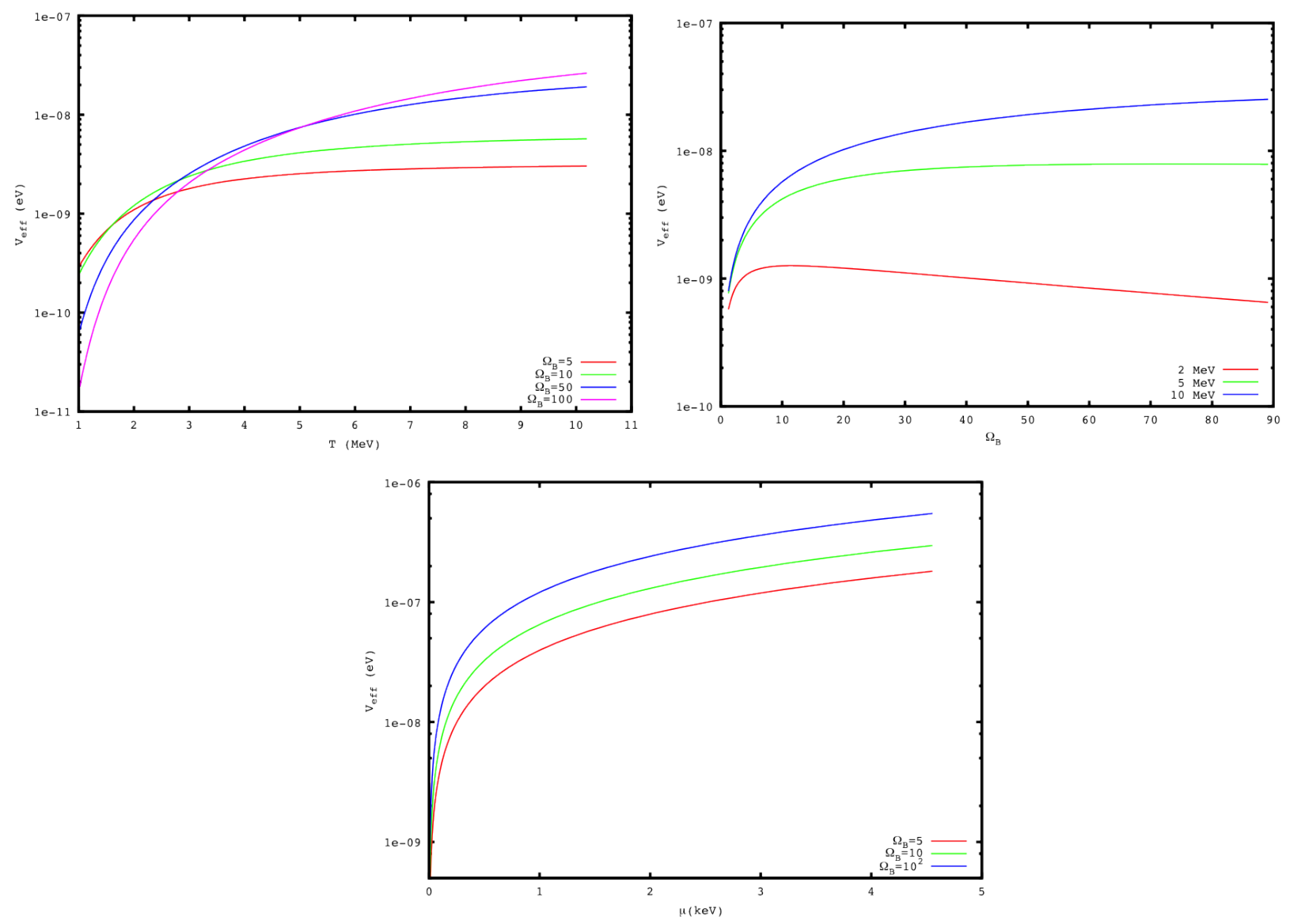

Fig. 3. - Neutrino effective potential in moderate above critical magnetic field regime as a function of temperature (T) (left-hand figure above), magnetic field $\left(\Omega_{B}\right)$ (right-hand figure above), chemical potential $(\mu)$ (figure below) and neutrino energy $\left(\mathrm{E}_{\nu}\right)$. All plots are obtained for a neutrino energy of $10 \mathrm{MeV}$. 

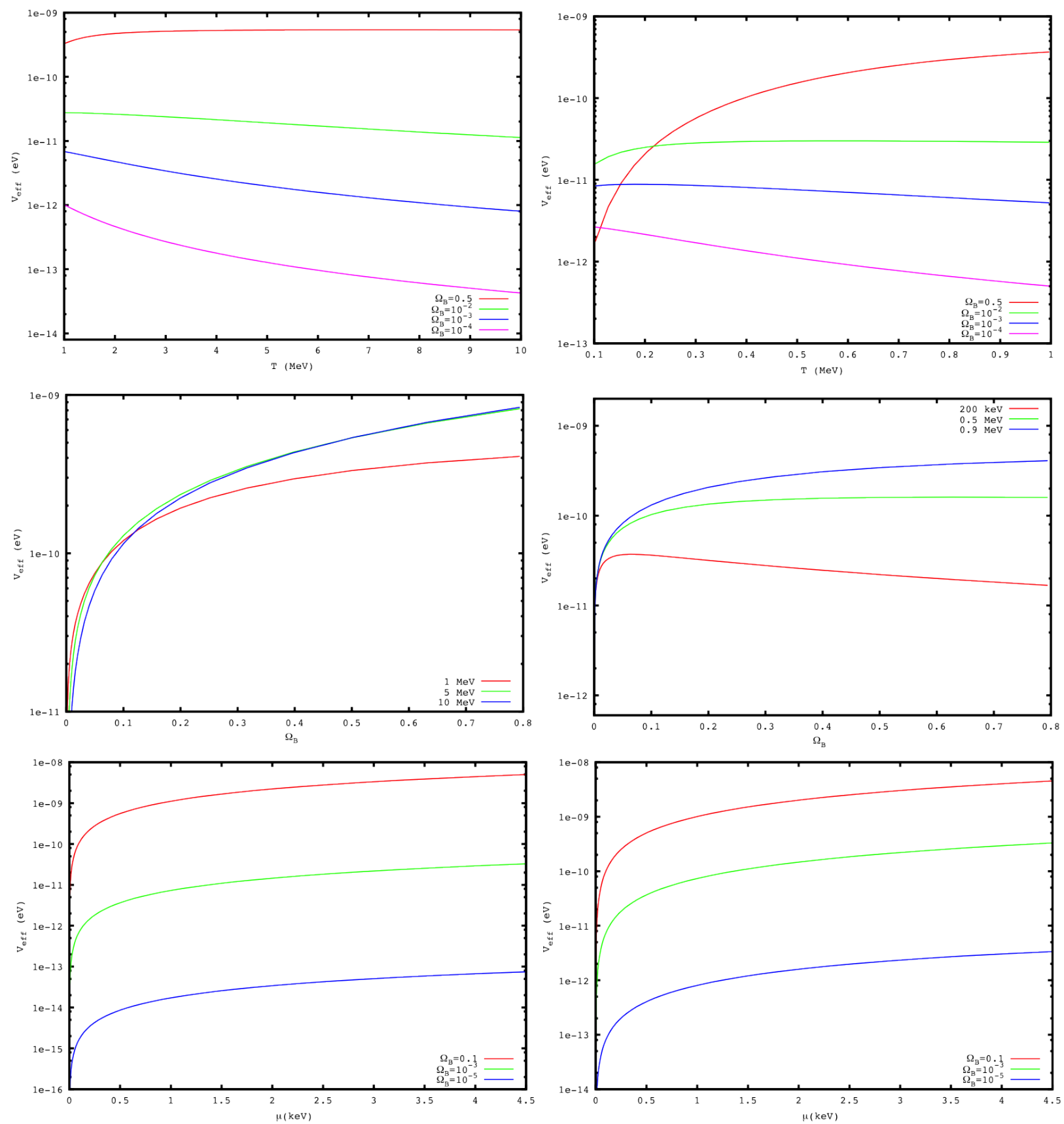

Fig. 4. - Neutrino effective potential in moderate below critical magnetic field regime as a function of temperature (T) (top), magnetic field $\left(\Omega_{B}\right)$ (middle), chemical potential ( $\mu$ ) (bottom). In the left column a neutrino energy $E_{\nu}=10 \mathrm{MeV}$ was used whereas the right column was plotted for $E_{\nu}=10 \mathrm{GeV}$ 

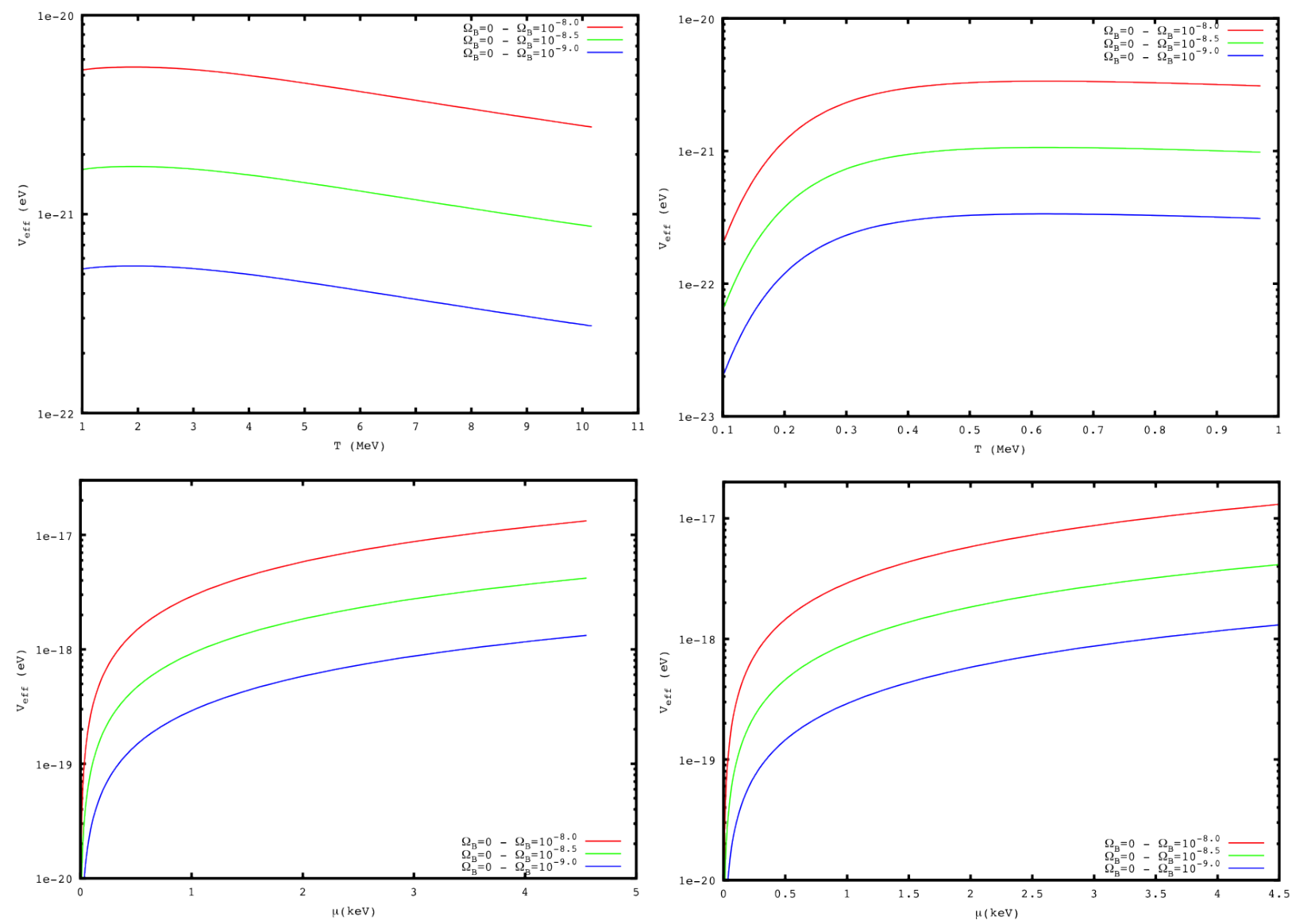

Fig. 5.- Neutrino effective potential in weak magnetic field regime as a function of temperature (T) (above) and chemical potential ( $\mu$ ) (below). In the left column a neutrino energy $E_{\nu}=10 \mathrm{MeV}$ was used whereas the right column was plotted for $E_{\nu}=10 \mathrm{GeV}$. 

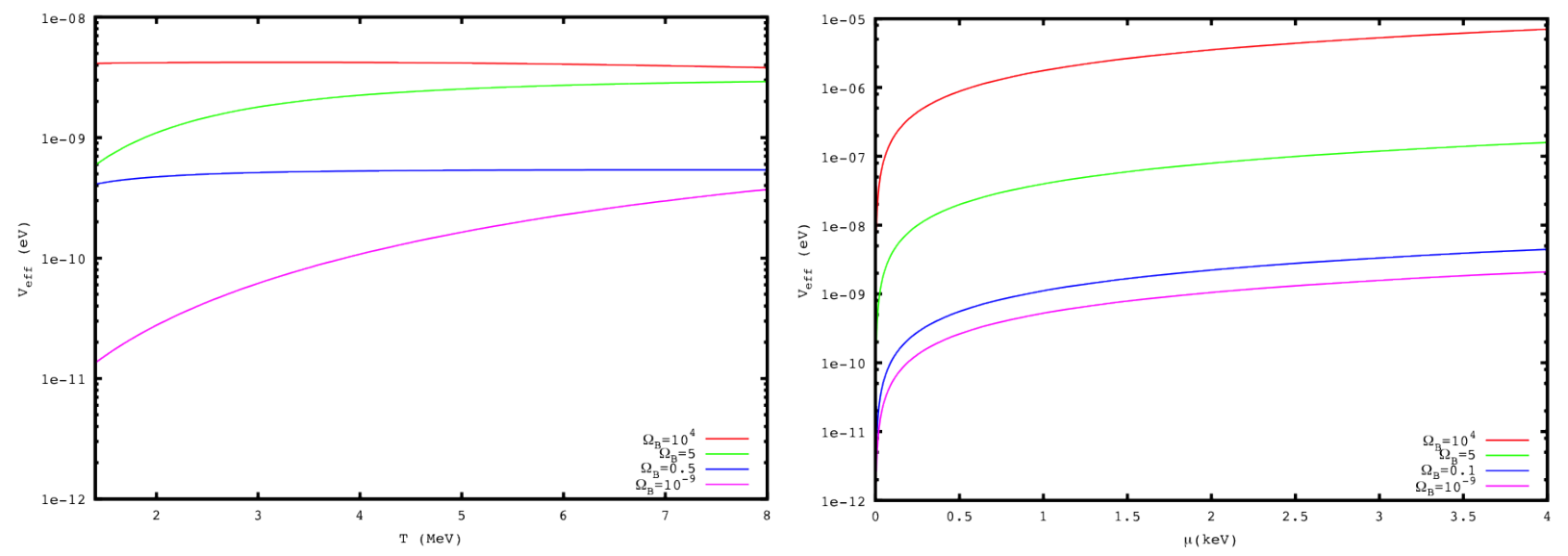

Fig. 6.- Neutrino effective potential as a function of temperature (left) and chemical potential (right) for fixed values of magnetic field in the strong $\left(10^{4} B_{c}\right)$, moderate ( 5 and $0.5 B_{c}$ for above and below, respectively) and weak $\left(10^{-9} B_{c}\right)$ regime.
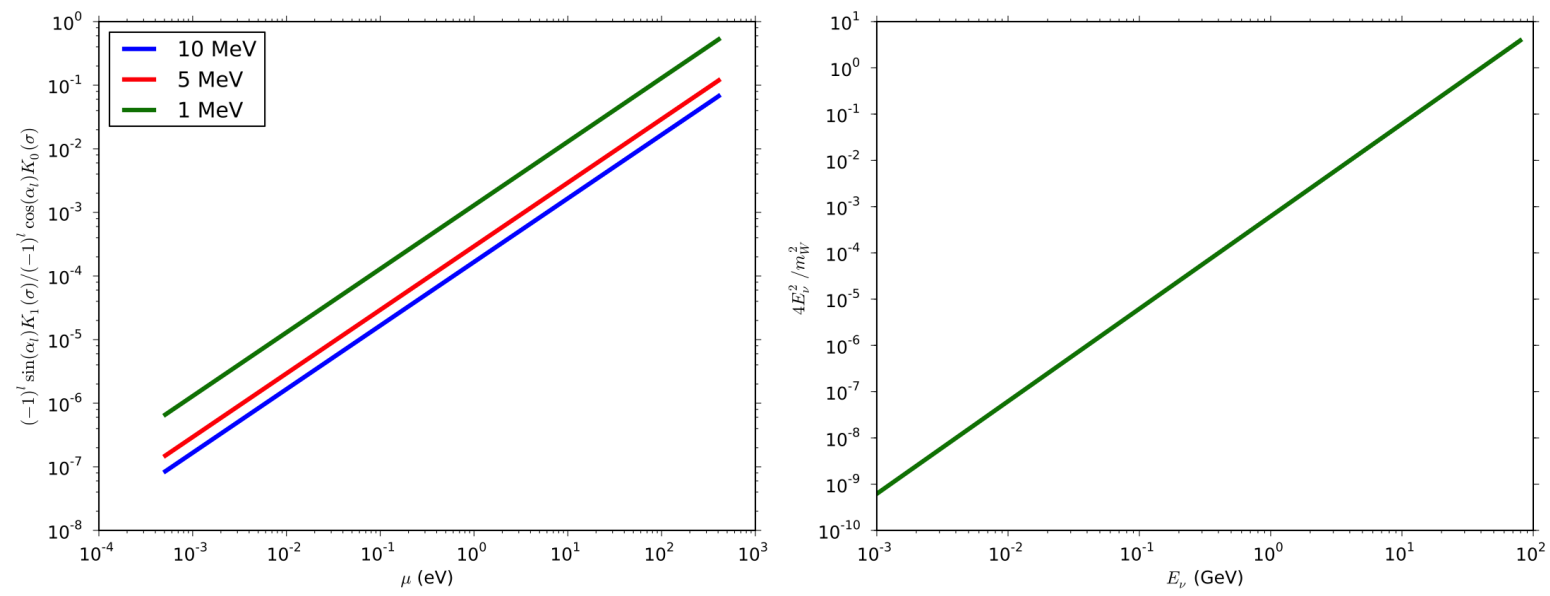

Fig. 7.- Contribution of the terms of order $\mathrm{m}_{W}^{-4}$ in the neutrino effective potential. 

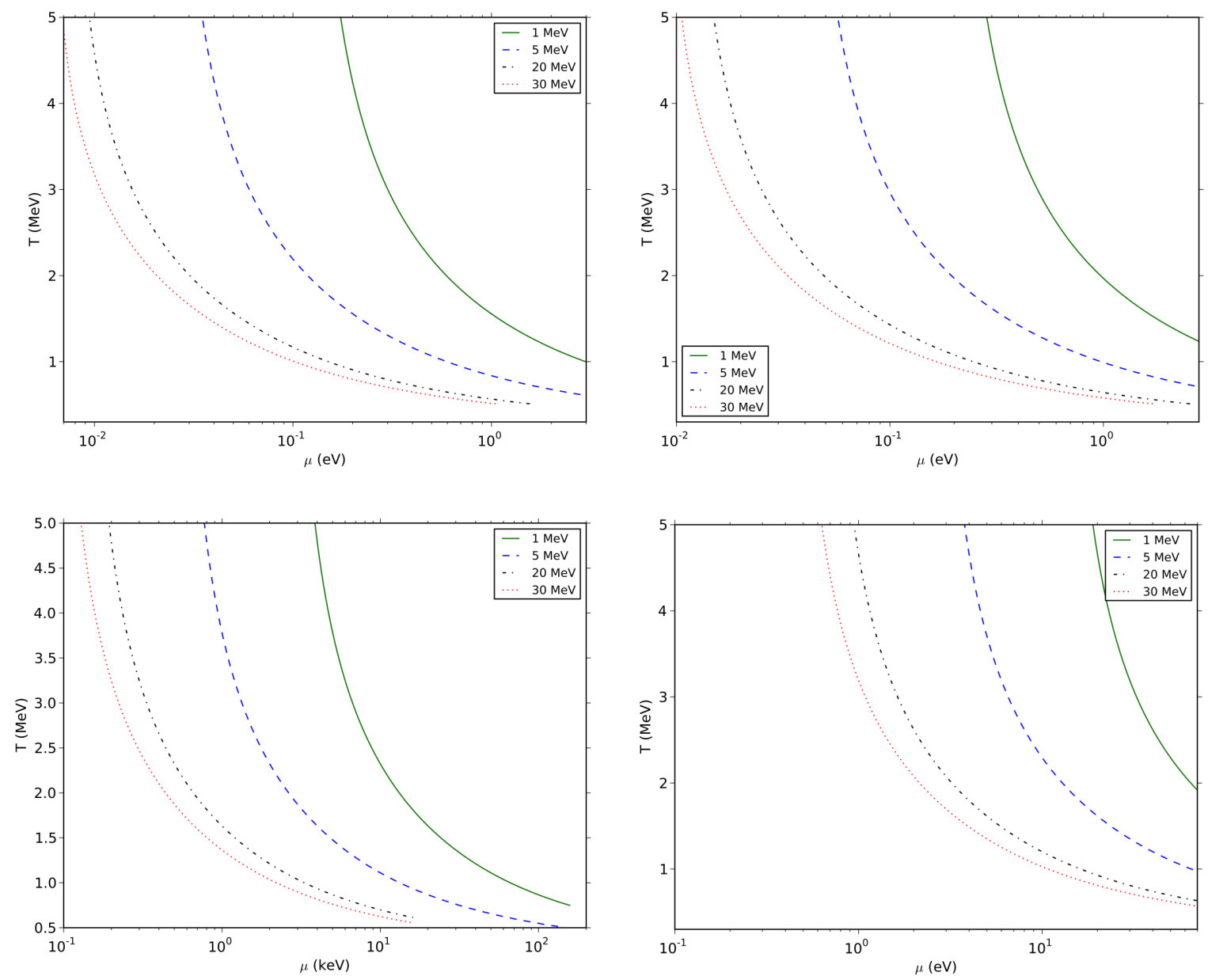

Fig. 8. - Contour plots of temperature $(\mathrm{T})$ and chemical potential $(\mu)$ as a function of thermal neutrino energy for which the resonance condition is satisfied. We have applied the neutrino effective potential with moderate magnetic field $\left(\Omega_{B}=10\right)$ and used the best fit values of the two; solar (left-hand figure above), atmospheric (right-hand figure above), and accelerator (left-hand figure below), and three (left-hand figure below) neutrino mixing. 

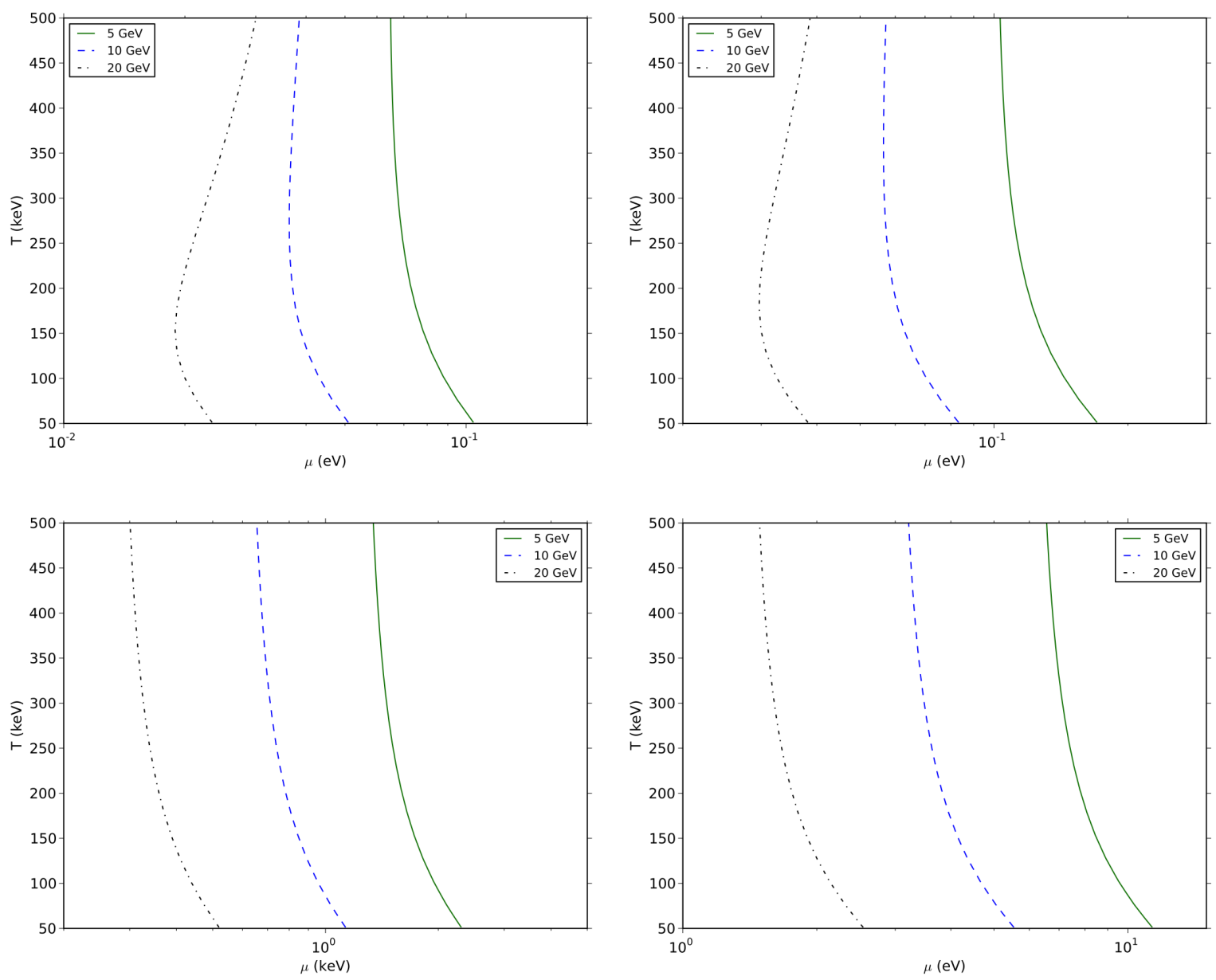

Fig. 9.- Contour plots of temperature (T) and chemical potential $(\mu)$ as a function of quasi-thermal neutrino energy for which the resonance condition is satisfied. We have applied the neutrino effective potential with moderate magnetic field $\left(\Omega_{B}=10^{-4.3}\right)$ and used the best fit values of the two; solar (left-hand figure above), atmospheric (right-hand figure above), and accelerator (left-hand figure below), and three (left-hand figure below) neutrino mixing. 

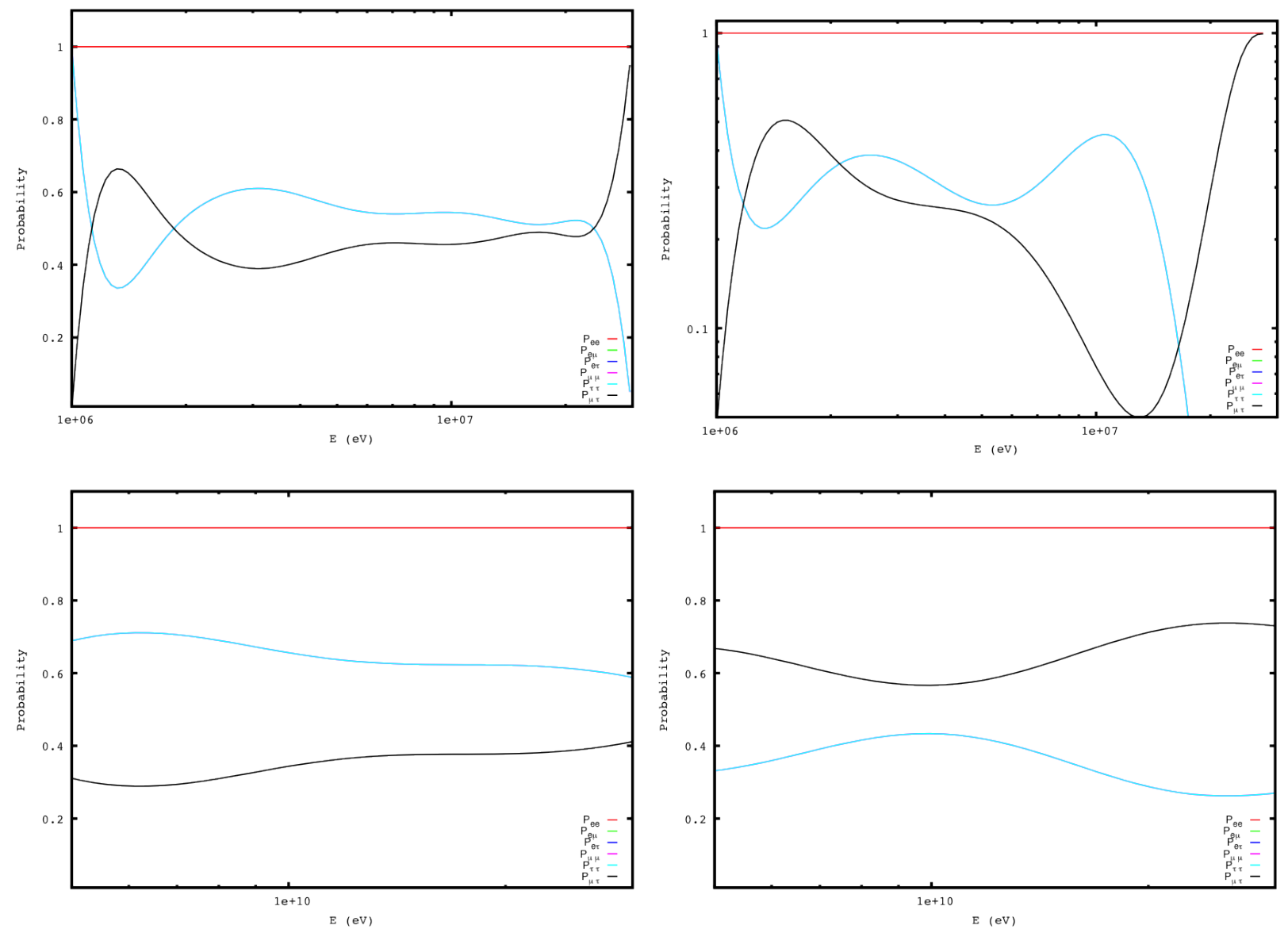

Fig. 10.- Oscillation probabilities of thermal MeV (figures above) and sub-photospheric (figures below) neutrinos as a function of neutrino energy are plotted. For thermal neutrinos we have used the values of magnetic field $10 B_{c}$ and the fireball radii $10^{7} \mathrm{~cm}$ (left figure) and $10^{8} \mathrm{~cm}$ (right figure) whereas for sub-photospheric neutrinos we have used the values $\mathrm{B}=10^{-4.3} B_{c}$ and $10^{11} \mathrm{~cm}$ (left figure) and $10^{12} \mathrm{~cm}$, respectively. 

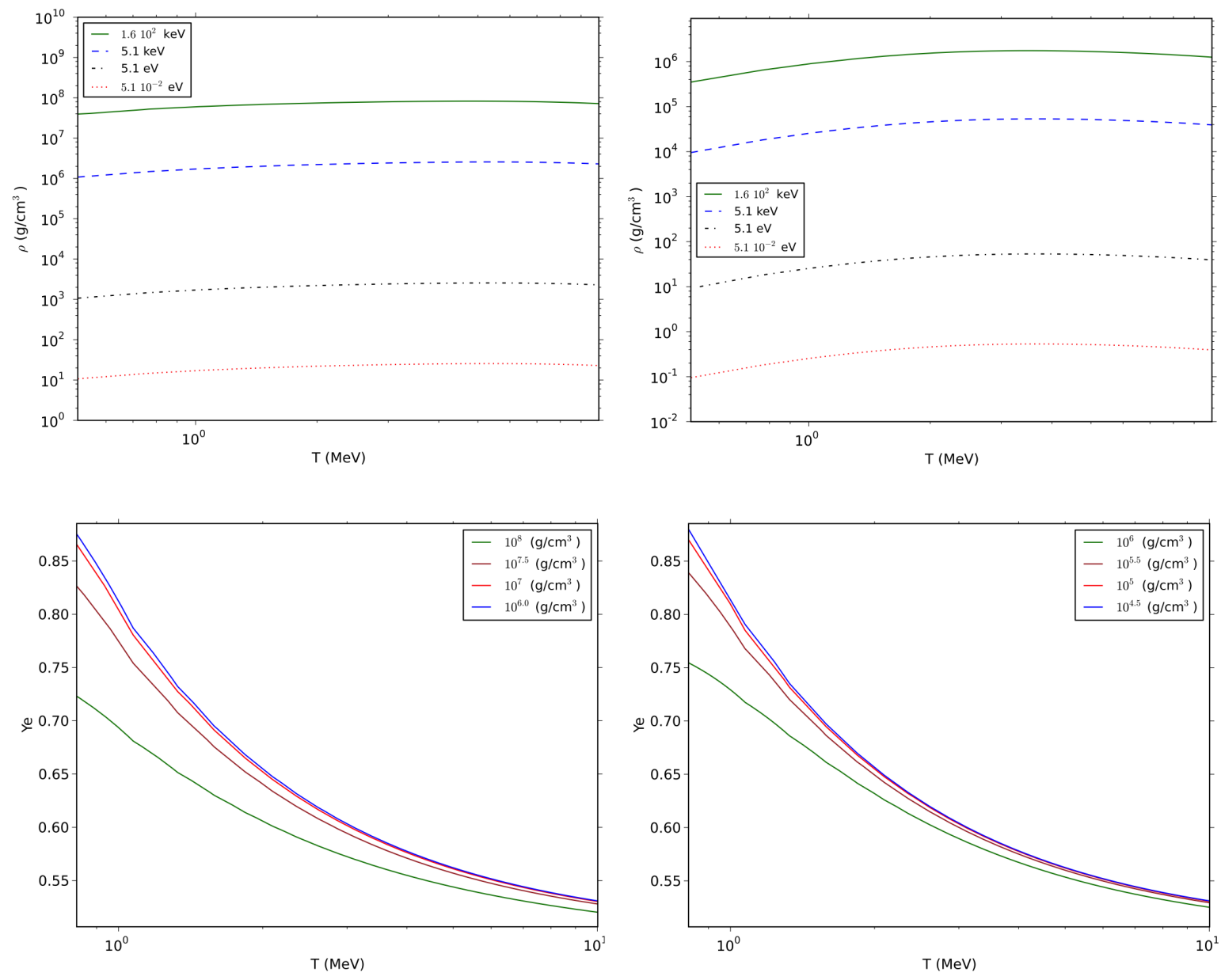

Fig. 11. - Contour plots of baryon density (above) and Ye (below) as a function of temperature for which the equilibrium condition is established. We use the values of temperature and chemical potential in the resonance condition range and moderate field limit: $50 B_{c}$ (left-hand figures) and $0.1 B_{c}$ (right-hand figures). 

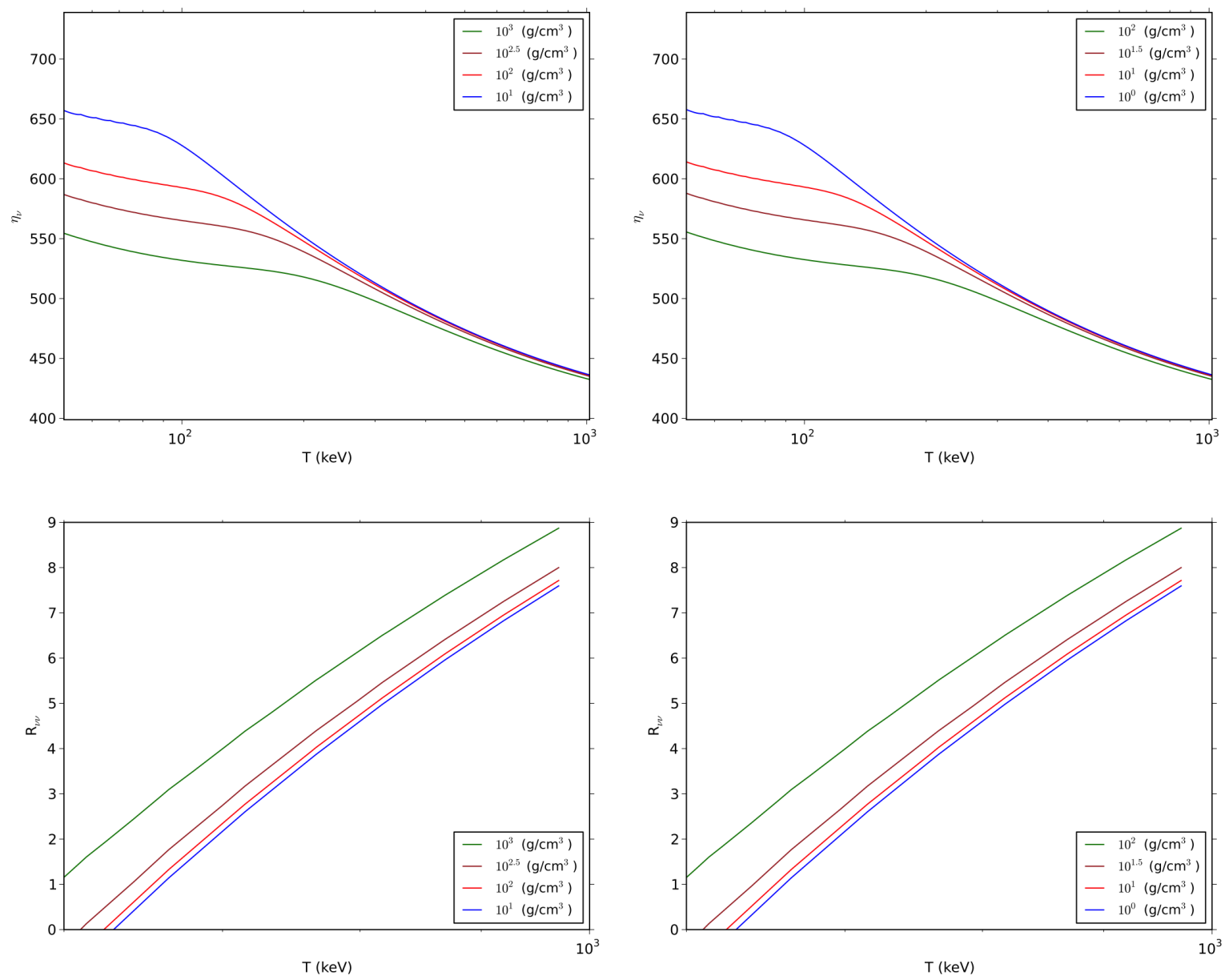

Fig. 12.- Contour plots of the critical value of baryon load parameter $\left(\eta_{\nu}\right)$ (above) and number of expected events $\left(R_{\nu \nu}\right)$ (below) as a function of and temperature (T) (Meszaros \& Rees 2000). We use the values of temperature and chemical potential in the resonance condition range and moderate field limit: $10^{-4.3} B_{c}$ (left-hand figures) and $10^{-5.3} B_{c}$ (right-hand figures). The number of events is calculated for $\mathrm{z}=0.1$ 